\title{
Impact of magnetic transition on Mn diffusion in $\alpha$-iron: Correlative state-of-the-art theoretical and experimental study
}

\author{
Omkar Hegde $\oplus^{1},{ }^{1, *}$ Vladislav Kulitckii $\odot,{ }^{2}$ Anton Schneider $\odot,{ }^{3, \dagger}$ Frédéric Soisson $\odot{ }^{3},{ }^{3}$ Tilmann Hickel $\odot,{ }^{1}$ Jörg Neugebauer, ${ }^{1}$ \\ Gerhard Wilde, ${ }^{2}$ Sergiy Divinski ${ }^{2},{ }^{2,4}$ and Chu-Chun Fu ${ }^{3}{ }^{3, \ddagger}$ \\ ${ }^{1}$ Max-Planck-Institut für Eisenforschung, Düsseldorf 40237, Germany \\ ${ }^{2}$ Institute of Materials Physics, Westfälische Wilhelms-University of Münster, Germany \\ ${ }^{3}$ Université Paris-Saclay, CEA, Service de Recherches de Métallurgie Physique, 91191 Gif-sur-Yvette, France \\ ${ }^{4}$ Samara National Research University, Moskovskoye Shosse 34, Samara 443086, Russia
}

(Received 14 July 2021; accepted 25 October 2021; published 10 November 2021)

\begin{abstract}
An accurate prediction of atomic diffusion in Fe alloys is challenging due to thermal magnetic excitations and magnetic transitions. We investigate the diffusion of $\mathrm{Mn}$ in bcc Fe using an effective interaction model and first-principles based spin-space averaged relaxations in magnetically disordered systems. The theoretical results are compared with the dedicated radiotracer measurements of ${ }^{54} \mathrm{Mn}$ diffusion in a wide temperature range of 773 to $1173 \mathrm{~K}$, performed by combining the precision grinding (higher temperatures) and ion-beam sputtering (low temperatures) sectioning techniques. The temperature evolution of Mn diffusion coefficients in bcc iron in theory and experiment agree very well and consistently reveal a reduced acceleration of Mn solute diffusion around the Curie point. By analyzing the temperature dependencies of the ratio of Mn diffusion coefficients to self-diffusion coefficients we observe a dominance of magnetic disorder over chemical effects on high-temperature diffusion. Therefore, the missing acceleration mainly reflects an anomalous behavior of the Mn solute in the magnetically ordered low-temperature state of the Fe host, as compared to other transition metals.
\end{abstract}

DOI: 10.1103/PhysRevB.104.184107

\section{INTRODUCTION}

Atomic diffusion plays a central role dictating the kinetics of numerous physical processes in solids, such as surface and interfacial segregation, precipitation, and phase transitions. Iron-based alloys, being the basis of steels, are certainly one of the most studied systems from both theoretical and experimental points of view. Experimental data for solute and solvent diffusion in iron alloys are usually known only at relatively high temperatures (above $750 \mathrm{~K}$ ) [1-13], due to the general limitations of a standard radiotracer technique [14]. On the other hand, a quantitative model of the diffusion coefficients as functions of temperature in these systems is not obvious, since the effects of thermal magnetic excitations and magnetic order-disorder transition need to be properly described.

Indeed, thermodynamic and kinetic properties of Fe-based alloys may strongly be influenced by magnetism. Most

\footnotetext{
*Corresponding author: o.hegde@mpie.de

${ }^{\dagger}$ Present address: Engineering Physics Department, University of Wisconsin, Madison WI 53706, USA

${ }^{\ddagger}$ Corresponding author: chuchun.fu@cea.fr
}

Published by the American Physical Society under the terms of the Creative Commons Attribution 4.0 International license. Further distribution of this work must maintain attribution to the author(s) and the published article's title, journal citation, and DOI. Open access publication funded by the Max Planck Society. prominently, the magnetic transition in $\mathrm{Fe}$ is correlated with a relative (with respect to an Arrhenius-type temperature dependence) acceleration of the self- and solute diffusion coefficients when the Curie temperature $T_{\mathrm{C}}$ is approached and the ferromagnetic ordering is vanishing (or, equivalently, a retardation of diffusion in magnetically ordered $\mathrm{Fe}$ with respect to a low-temperature extrapolation of the data for its paramagnetic state) $[1-4,6-9,15,16]$. It was also shown that the concentration dependence of the magnetic transition in Fe-Co alloys is related to the body-centered-cubic (bcc) to face-centered-cubic (fcc) transition [17-19]. Further, previous studies have shown that magnetism in $\mathrm{Fe}-\mathrm{Cr}$ alloys has a crucial impact on the mixing enthalpy and induces an asymmetry in the mutual solubility of $\mathrm{Fe}$ and $\mathrm{Cr}$ at low temperature [20,21].

This paper is mainly focused on bcc Fe-Mn alloys, for which such effects are much less understood. Already pure bulk Mn shows a complex magneto-structural phase diagram [22,23] and strong magneto-elastic coupling effects are also present in Fe-Mn alloys [24]. According to phase diagrams $[25,26]$, the stability domain of bcc Fe-Mn alloys is limited to dilute alloys $(<5$ at $\% \mathrm{Mn})$. The magnetism of $\mathrm{Mn}$ solutes in bcc Fe is complex, which makes bcc Fe-Mn alloys even in the Fe-rich part an interesting case to study [27,28]. Due to the presence of a high Mn majority-spin electronic density around the Fermi level, its magnetic interaction is highly sensitive to minor changes of the Mn local environment [28]. For example, an isolated $\mathrm{Mn}$ atom in bcc Fe has its magnetic moment antiparallel to the Fe moments in the ground state, but the parallel configuration is another energy 
minimum that becomes dominant at larger Mn concentrations in $\mathrm{Fe}[27,28]$. Here, we show that this particularity of $\mathrm{Mn}$ in $\mathrm{Fe}$ has a qualitative impact on the diffusion properties around $T_{\mathrm{C}}$.

Even the self-diffusion in iron via the vacancy mechanism is still a topic of active research [16,28-32]. A number of approaches were proposed to describe the temperature dependence of the self-diffusion coefficient in the whole temperature range of bcc $\mathrm{Fe}$ by a single equation $[1,9,33]$. Based on the theoretical model of Girifalco [34], which analyzed the effects of ordering on diffusion in $\beta$ brass, Ruch et al. [33] have fitted the temperature dependence of the self-diffusion coefficient in $\alpha$-Fe as

$$
\begin{aligned}
D(T) & =D_{0}^{\mathrm{PM}} \exp \left[-\frac{E_{a}^{\mathrm{mag}}(T)}{k_{B} T}\right] \\
\text { with } \quad E_{a}^{\mathrm{mag}}(T) & =E_{a}^{\mathrm{PM}}\left(1+\alpha(M(T) / M(0))^{2}\right)
\end{aligned}
$$

where the pre-exponential factor $D_{0}^{\mathrm{PM}}$ and the activation energy of diffusion $E_{a}^{\mathrm{PM}}$ belong to the paramagnetic state. $M(T)$ is the magnetization at a temperature $T$, yielding a maximum of the activation enthalpy in the ferromagnetic limit as $E_{a}^{\mathrm{FM}}=E_{a}^{\mathrm{PM}}(1+\alpha)$. Therefore, the dimensionless constant $\alpha$ describes the effect of magnetic ordering on the activation energy of diffusion.

The solute diffusion in $\mathrm{Fe}$ across the Curie point has so far only been addressed in a few theoretical studies [16,28,29,31]. In experiment, however, a behavior similar to the representation in Eq. (2) was observed for a number of solutes, e.g., for $\mathrm{Co}[5,35], \mathrm{Cr}[36]$, and $\mathrm{Ni}[35]$ in bcc Fe, when diffusion has been measured over sufficiently large temperature intervals. Comparing the magnetic moment of a solute impurity in the bcc Fe matrix and the value of $\alpha$ used to fit the experimental data, empirically yields a linear correlation, i.e., higher magnetic moments seem to correspond to higher $\alpha$ values [9,37].

Diffusion of $\mathrm{Mn}$ solutes in $\mathrm{Fe}$ was previously measured by several researchers, see Fig. 1. The results of Nohara and Hirano [38] and Lübbehusen [15] measured in $\alpha$-Fe agree with the independent data of Kirkaldy et al. [39] reported for $\delta$-Fe. A formal fit of these data using the Ruch model would predict $\alpha_{\mathrm{Mn}} \approx 0$. However, since the measurements in Refs. $[15,38]$ were performed at relatively high temperatures near the Curie temperature of $\alpha$-Fe the understanding of the influence of the magnetic transition on Mn diffusion is currently limited. Furthermore, a large scatter of the data measured for bcc Fe in the paramagnetic state, Fig. 1, demands further clarification.

While there are indications that Mn does not follow the above mentioned trend for $\alpha$ values, a thorough study is missing so far. Studies based on density functional theory (DFT) have been reported in the literature by Messina et al. [41] and Versteylen et al. [42]. In these papers, detailed studies on Mn-vacancy binding energies, migration barriers for the Le Claire's model and activation energies have been reported for the ferromagnetic state. However, paramagnetic Mn-vacancy binding energies and migration barriers were not evaluated in these studies. Though vacancy activation energies in the paramagnetic state were determined using ferromagnetic activation energies and $\alpha$ (since $E_{a}^{\mathrm{FM}}=E_{a}^{\mathrm{PM}}(1+\alpha)$ ), Messina et al. assumed the $\alpha$ value of Fe self-diffusion for Mn impurity

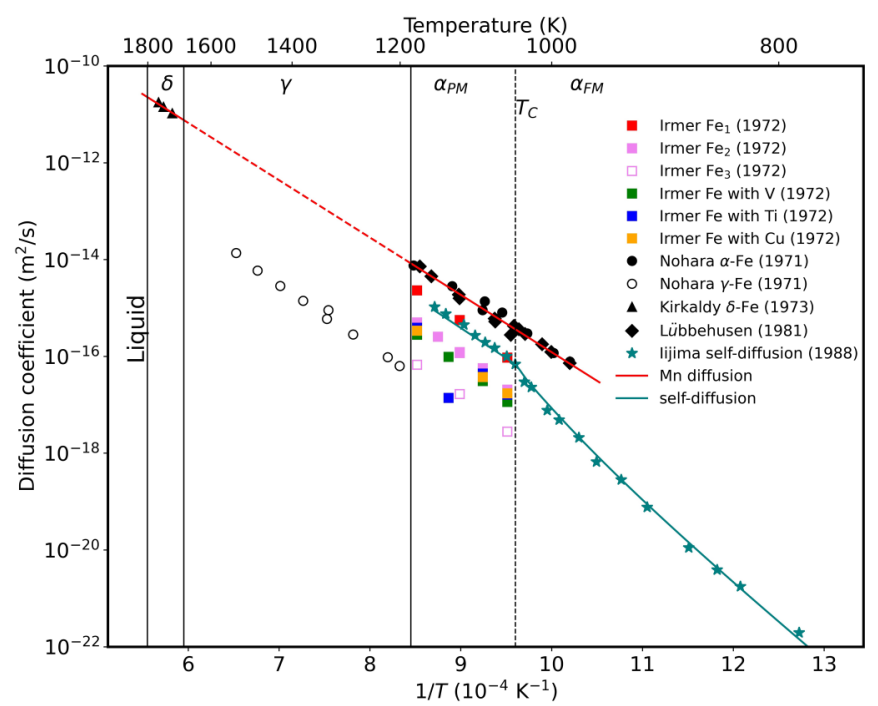

FIG. 1. Literature data on Mn diffusion in $\alpha$-Fe. A formal fit of the data of Lübbenhusen [15] and Nohara and Hirano [38] using the Ruch model [33] (solid line) would predict $\alpha \approx 0$. For completeness, the results of the measurements of Irmer and Feller-Kniepmeier (squares) [40] and and Kirkaldy et al. (triangles) [39] are shown. For reference, the experimental data for Fe self-diffusion in $\alpha$-Fe measured by Iijima et al. [4] are shown, too (stars). Irmer and FellerKniepmeier measured Mn diffusion in different kinds of pure $\mathrm{Fe}$ and $\mathrm{Fe}$ alloys, for further details please refer to the original paper [40]. The vertical lines indicate the phase transition temperatures.

diffusion in $\mathrm{Fe}$, whereas Versteylen et al. did not explain how they determined $\alpha$ value and cited their unpublished work. In this paper, we therefore investigate to what extent the nonArrhenius acceleration of diffusion near $T_{c}$ described above [10-13] is also observed for $\mathrm{Mn}$ in Fe or if it is substantially reduced as compared to $\mathrm{Fe}$ self-diffusion.

To this end, we perform radiotracer diffusion experiments that give access to Mn diffusion coefficients at lower temperatures than reported in the literature. This allows a more accurate study of modifications of the solute diffusion coefficients due the magnetic transition at the Curie temperature. Along with the experiments, we deploy two complementary theoretical approaches to compute vacancy formation and migration energies in the presence of a Mn solute next to a vacancy.

Based on the method suggested by Hegde et al. [32], first-principles DFT calculations are combined with spinspace averaged (SSA) forces [43], to account for the effect of magnetic disorder on atomic relaxations and the subsequent impact on the vacancy activation energies. We analyze whether the significant effect of relaxations on the vacancy energetics observed in pure $\mathrm{Fe}$ also applies to the vacancy formation and migration energies in Fe-Mn. While such SSA relaxations allow for a direct and accurate computation of the energies in the paramagnetic regime, an interpolation scheme like the Ruch model (2) is used to extract the temperature evolution of diffusion coefficients between the fully ferromagnetic and the fully paramagnetic regimes.

The second approach has been proposed by Schneider et al. [44] and enables a continuous prediction of dif- 
fusion properties versus temperature, including explicitly spin and atomic variables. Adopting a DFT-based effective interaction model (EIM) coupled with on-lattice Monte Carlo (MC) simulations, the self- and $\mathrm{Cu}$ diffusion in bcc Fe was considered to illustrate the ability of the methodology to predict diffusion properties. In the present paper, we apply the same methodology to the diffusion of Mn solutes. While this model does not require a scheme to interpolate temperature, the accuracy of the results depends on the parametrization of the EIM.

The comparison of these two complementary approaches and the tracer diffusion experiments guarantees the robustness of the result and will provide new insights concerning a potential diffusion anomaly of $\mathrm{Mn}$ in bcc Fe. The first section of the paper is dedicated to the methodology, including details concerning experiments, DFT calculations, the EIM approach, the study of diffusion, and Monte Carlo simulations. Then the approach is applied in the following section to the study Mn diffusion in bcc Fe. Finally, the agreement with experimental results is discussed.

\section{METHODOLOGY}

\section{A. Density functional theory calculations}

In this work, DFT calculations are performed for (1) the SSA based relaxations and (2) for parametrizing the EIM model. The details are given in the following:

\section{SSA relaxation method for magnetic disorder}

To determine the vacancy formation energy, binding energy, and migration barriers of different exchanges in the neighbourhood of the vacancy, DFT calculations have been employed using a $3 \times 3 \times 3$ supercell of $\alpha$-Fe with a single vacancy and a single $\mathrm{Mn}$ atom. The novelty of the present approach is to fully account for the structural relaxation effects in the disordered magnetic state [32]. The scheme is based on the SSA [45] technique, which assumes that the magnetic degrees of freedom in the paramagnet vary much faster than the atomic degrees of freedom, viz., the fast magnetic degrees adiabatic approximation.

Therefore, the Hellmann-Feynman forces on the atoms for each lattice position need to be averaged over a large number of disordered magnetic configurations. The subsequent structural optimization is carried out using these averaged forces and a new geometrical structure is obtained, which is again experiencing the magnetic disorder. This procedure is performed till the SSA forces converge to the required accuracy. To automatically combine the different simulation steps and tools, we employ the integrated development environment (IDE) pyiron [46].

The presence of Mn in the vicinity of the vacancy yields the vacancy-Mn binding energy

$$
E_{\text {binding }}=\left(E_{\mathrm{vac}}^{(N-1)}+E_{\mathrm{Mn}}^{(N-1)}\right)-\left(E_{\mathrm{vac}, \mathrm{Mn}}^{(N-2)}+E^{(N)}\right) .
$$

The energies on the right-hand side of the equation refer to supercells with both vacancy and $\mathrm{Mn}$, without defect, with a single vacancy and a single $\mathrm{Mn}$ atom in the Fe bulk, respectively. The number of $\mathrm{Fe}$ atoms in each case is given by the superscript. According to this definition, a positive binding energy would mean an attractive interaction between the vacancy and Mn.

The migration barriers are computed by the activationrelaxation technique (ART) [47]. The migration energy corresponding to the transition (trans) state is defined as

$$
E_{m}=E_{\text {trans }}-E_{\mathrm{vac}}
$$

where $E_{\text {trans }}$ stands for total energy of the supercell in the transition state.

A supercell of 54 atoms $(3 \times 3 \times 3$ cell $)$ is used for ferromagnetic bulk calculations and the collinear magnetically disordered state for this supercell is realized by a special quasirandom structure (SQS) [48]. The experimental lattice parameter [49] of $2.86 \AA$ is used for the ferromagnetic structures of both $\mathrm{Fe}$ and FeMn supercells containing one Mn atom. For the paramagnetic Fe and FeMn SQSs an experimental lattice parameter [49] of $2.90 \AA$ is used, therewith taking thermal expansion into account. Structural optimization is carried out with SxExtOpt [50] and a total of eight different magnetic structures are used for spin-space averaging. SxExtOpt is an efficient external optimizer that performs on-the-fly parametrization of the Hessian.

These DFT calculations are carried out with the SPHInX [51] package, within the Perdew-Burke-Ernzerhof (PBE) generalized-gradient approximation (GGA) [52,53], using the projector augmented wave (PAW) [54,55] formalism. An energy cutoff of $600 \mathrm{eV}$ and a Monkhorst-Pack $k$-point mesh of $6 \times 6 \times 6$ is used, after ensuring that the error due to convergence is less than $1 \mathrm{meV} /$ atom. All calculations are performed with Fermi-Dirac smearing, with a width of $0.1 \mathrm{eV}$ (electronic temperature close to $T_{\mathrm{C}}=1041 \mathrm{~K}$ ). The convergence criterion for total energy in electronic relaxation is $10^{-5} \mathrm{eV}$ and the convergence criterion for forces in ionic relaxation is $0.015 \mathrm{eV} / \AA$.

Spins are not constrained for the ferromagnetic calculations, while spin constrained calculations are carried out for the paramagnetic calculations. The magnitude of the spin constrained moments is carefully optimized with respect to the total energy. In the ferromagnetic Fe matrix, the Mn atom in the dilute limit couples antiferromagnetically to the neighboring Fe atoms and its magnetic moment is $2.05 \mu_{B}$, consistent with previous results [28]. In the paramagnetic, i.e., magnetically disordered state, the magnitude of the moment of Mn atom is $1.8 \mu_{B}$. Since the local moments are larger for the atoms in the immediate vicinity of the vacancy, these changes have been determined by a moment-energy optimization for the first nearest neighbours atoms of the vacancy.

The magnetic entropy part of the vacancy formation and migration free energy does not yield a contribution in the SSA approach, since it vanishes in the limiting cases, i.e., in the ferromagnetic and paramagnetic regime [44].

\section{Parameterization of the effective interaction models}

Although a full description of DFT calculations for paramaterizing EIM models is provided in Ref. [28], the main computational details are repeated in this section. The calculations are also performed using the PAW method [54,55] and the PBE exchange-correlation functional, but employing the VASP (Vienna Ab initio Simulation Package) code [56-58]. 
All the calculations are spin-polarized. $3 d$ and $4 s$ electrons are considered as valence electrons. The plane-wave basis cutoff is set to $400 \mathrm{eV}$. Atomic magnetic moments are obtained by a charge and spin projection onto the PAW spheres [57,58] as defined by the PAW potentials.

The $k$ point grids are supercell-dependent chosen such that they are equivalent to a bcc cubic unit cell with a $16 \times 16 \times 16$ shifted grid, following the Monkhorst-Pack scheme [59]. The Methfessel-Paxton [60] broadening scheme with $0.1 \mathrm{eV}$ width is used. The convergence threshold for the electronic selfconsistency loop is $10^{-6} \mathrm{eV}$ and atomic relaxations at constant volume are performed down to a maximum residual force of $0.02 \mathrm{eV} / \AA$. We have verified that the magnetic structures and cluster formation energies are converged such that the resulting error bars for energy differences and magnetic moments of Fe and $\mathrm{Mn}$ are respectively $0.02 \mathrm{eV}, 0.01 \mu_{\mathrm{B}}$ and $0.1 \mu_{\mathrm{B}}$. These are mainly associated to the convergence of the plane-wave energy cutoff and the $k$-grid density.

In addition to the details given in Ref. [61], these calculations were performed at constant volume on 128-sites supercells $(4 \times 4 \times 4$ unit cells $)$ with a lattice constant of $2.83 \AA$ for pure FM bcc Fe optimized using the present computational details.

Though the computational details such as smearing schemes, energy cutoff are slightly different for SSA and EIM, careful convergence tests are performed for both the methods. In the ferromagnetic state, the resulting Mn-vacancy binding energies, vacancy formation energies and migration energies deviate by less than $0.05 \mathrm{eV}$ between the methods. Also, the literature values [41,42] for these energies in the ferromagnetic state are within an interval of $0.05 \mathrm{eV}$ compared to the two methods (for example, see Table III). Therefore, the deviations of the computed energies due to different computational details, including smearing schemes, can be regarded as sufficiently small.

\section{B. Effective interaction model (EIM)}

The EIM used in this study consists of a magnetic part with a Landau-Heisenberg form as in Refs. [44,62-65], which allows to account for both longitudinal and transversal excitations of spins. The Hamiltonian has the following formal expression:

$$
\begin{aligned}
H= & \sum_{i}^{N} A_{i}^{(S)} M_{i}^{2}+B_{i}^{(S)} M_{i}^{4}+\sum_{i}^{N} \sum_{n}^{P} \sum_{j}^{Z_{n}} J_{i j}^{(n)} \mathbf{M}_{i} \cdot \mathbf{M}_{j} \\
& +\sum_{i}^{N} \sum_{n}^{P} \sum_{j}^{Z_{n}} V_{i j}^{(n)} \sigma_{i} \cdot \sigma_{j}
\end{aligned}
$$

where $N$ is the total number of atoms, $P$ is the maximum range of interactions in terms of neighbor shells, $Z_{n}$ is the coordination number of each neighboring shell, $\mathbf{M}_{i}$ is a vector representing the magnetic moment of the $i$ th atom, $M_{i}$ is its magnitude. $V_{i j}^{(n)}$ and $J_{i j}^{(n)}$ represent respectively the chemical pair-interaction and the magnetic exchange-coupling parameters between atoms $i$ and $j$, at a range $n$. As shown in the Appendix, these interaction parameters $V_{i j}^{(n)}$ and $J_{i j}^{(n)}$ depend on the chemical species of the $i$ th and the $j$ th atoms (for instance, the magnetic exchange-coupling parameter used to calculate the interaction between a Fe and a Mn atom at $2 n n$ distance is $\left.J_{\mathrm{Fe}-\mathrm{Mn}}^{(2)}\right) . \sigma_{i}$ is the occupation of the $i$ th site, which value is 1 if there is an atom on the site, and 0 if there is a vacancy. Note that since every pair interaction is counted twice, the $1 / 2$ factor is included in the pairwise interaction parameters $\left(V_{i j}^{(n)}\right.$ and $\left.J_{i j}^{(n)}\right)$ listed in Appendix. $A_{i}^{(S)}$ and $B_{i}^{(S)}$ are the magnetic on-site parameters of the $i$ th atom. The $S$ exponent designates the proximity of a vacancy. Its value is 1 if the $i$ th atom is nearest neighbor to a vacancy, 2 if it is next-nearest neighbor to a vacancy, and 0 otherwise. Their role is to prevent the divergence of the magnetic moment magnitudes caused by the spin longitudinal variations due to the Heisenberg-like terms. Please note that the Appendix also lists parameters with star exponents. These parameters are used instead of their counterparts without stars if the $i$ th atom is located in a saddle-point position.

This model was obtained in several steps: First, an EIM for pure iron in a bcc lattice was parameterized on DFT data (see Ref. [44] for details). We checked that the Curie temperature is correctly reproduced $\left(T_{\mathrm{C}}=1050 \mathrm{~K}\right.$, compared to the experimental [66] value of $1044 \mathrm{~K}$ ). Then, to include the presence of a vacancy $\left(\mathrm{EIM}^{V}\right)$, the on-site $A_{i}, B_{i}$, and $J_{i j}$ parameters are modified for atoms located at the first and second nearest-neighbor (1nn and $2 n n$ ) sites of the vacancy, as explained in Ref. [61]. Vacancy formation energies for distinct magnetic spin configurations around the vacancy predicted by DFT are successfully captured by this simple model [44]. In order to simulate the atomic migration, a second pure-iron derived EIM $^{S P}$ is constructed to describe the energetics of an $\mathrm{Fe}$ atom at a saddle-point position. In this case, the on-site and $J_{i j}$ parameters of the saddle point atom and their $1 n n$ and $2 n n$ atoms are modified based on DFT data. The atom-vacancy exchange barriers are then determined by the energy calculated using the $\mathrm{EIM}^{V}$ and the $\mathrm{EIM}^{S P}$. Note that this approach to determine barrier was successfully applied in previous studies using nonmagnetic interaction models [21,67,68].

For Mn-diffusion in bcc Fe, a Mn atom and a vacancy have been included in the iron system. Similarly, an EIM with all the atoms at lattice positions and another one with an atom (Fe or $\mathrm{Mn}$ ) at a saddle-point site are parameterized on DFT data on Mn-vacancy binding energies and atom-vacancy exchange barriers with various spin configurations. The numerical parameters of the various EIMs are given in Ref. [44] and in the Appendix (Sec. A).

Please note that the lattice vibrational entropies are not intrinsically accounted in the present EIM-Monte Carlo setup. The attempt frequencies are calculated separately by DFT, using the frozen phonons methodology $[69,70]$. For this purpose, we used VASP along with the Phonopy software [71]. The magnon-phonon effects [43] are therefore not considered. For the rest of the paper, results attributed to EIM refer to the above discussed method, unless otherwise specified.

\section{Diffusion coefficients}

The tracer diffusion coefficients can be expressed with Einstein's formula [72-74]

$$
D^{*}=\frac{\left\langle r^{2}\right\rangle}{6 t},
$$


where $\left\langle r^{2}\right\rangle$ and $t$ are the mean square displacement of the tracers and the corresponding physical time. For the self-diffusion case, it can also be written in terms of the equilibrium vacancy concentration $C_{\mathrm{vac}}$, determined by its formation magnetic free energy and the magnetic free energy barrier for the vacancyFe exchange $E_{m}^{\mathrm{mag}}$ (vacancy migration) at a given $T$ as $[14,75]$

$$
D_{F e *}^{F e}=a^{2} f_{0} C_{\mathrm{vac}} v \exp \left[-\frac{E_{m}^{\mathrm{mag}}(T)}{k_{B} T}\right] .
$$

Here, $a$ is the lattice constant, $f_{0}$ is the self-diffusion correlation factor, $v$ is the attempt frequency, and $k_{B}$ is the Boltzmann factor. Both magnetic and vibrational entropies are considered in the EIM-MC study, and the latter is calculated via DFT in the ferromagnetic state. Since the magnetic entropy is calculated in EIM-MC, we use the term "magnetic free energy" for vacancy formation, migration, and activation (also referred as vacancy formation energy, migration energy and activation energy, respectively). As mentioned in Sec II A1, though magnetic entropy is not explicitly calculated within the SSA approach, using the "magnetic free energy" term is also justified for the SSA results, since the SSA interpolates between the total energies in the limiting cases of ferromagnetic and paramagnetic states, where the magnetic entropy is known to be zero [44].

Similarly, the solute (Mn) tracer diffusion coefficient in Fe in the dilute limit can be written $[14,75]$ as

$$
D_{M n *}^{F e}=a^{2} f_{2} C_{\text {vac }}^{1 n n} \nu_{2} \exp \left[-\frac{E_{m, \mathrm{Mn}}^{\mathrm{mag}}(T)}{k_{B} T}\right],
$$

where $C_{\text {vac }}^{1 n n}$ is the equilibrium vacancy concentration at a $1 n n$ site of the solute, $\nu_{2}$ is the vacancy-Mn exchange attempt frequency, $f_{2}$ is the solute diffusion correlation factor and $E_{m, \mathrm{Mn}}^{\mathrm{mag}}$ is the magnetic free energy barrier for the vacancysolute exchange. In the present paper, the attempt frequencies $v$ are assumed to be equal, since $\mathrm{Fe}$ and Mn have very similar atomic mass. The superscript "mag" in the $E_{m}^{\mathrm{mag}}$ is dropped in the following sections, since it is now obvious that we refer to magnetic free energy barriers.

\section{Ratio of diffusion coefficients}

To quantify the diffusion of the solute atom relative to the diffusion of the host atom, the ratio of diffusion coefficients as a function of temperature is required. For the DFT based relaxation method, considering the vacancy-solute interaction up to $1 n n$ distance, the ratio is computed according to Le Claire's formulation [76]:

$$
\frac{D_{\mathrm{Mn}}}{D_{\mathrm{Fe}}}=\frac{f_{2}}{f_{0}} \frac{\omega_{2} \omega_{4}}{\omega_{0} \omega_{3}} \quad \text { with } \quad \omega_{i}=v e^{-\frac{E_{i}^{(i)}}{k_{B} T}},
$$

where $D_{\mathrm{Mn}}$ and $D_{\mathrm{Fe}}$ are the $\mathrm{Mn}$ and self-diffusion coefficients respectively, and $\omega_{i}$ are the jump frequencies (shown in Fig. 2).

First, we calculate migration barriers for different jumps of the vacancy. Since the $2 n n$ binding energies $E_{\text {binding }}$ are weak for both ferromagnetic and paramagnetic states, one can assume that the vacancy exchanges are not affected by the presence of the $\mathrm{Mn}$ atom beyond the 1nn shell. Moreover, since the paramagnetic calculations in the DFT-SSA approach

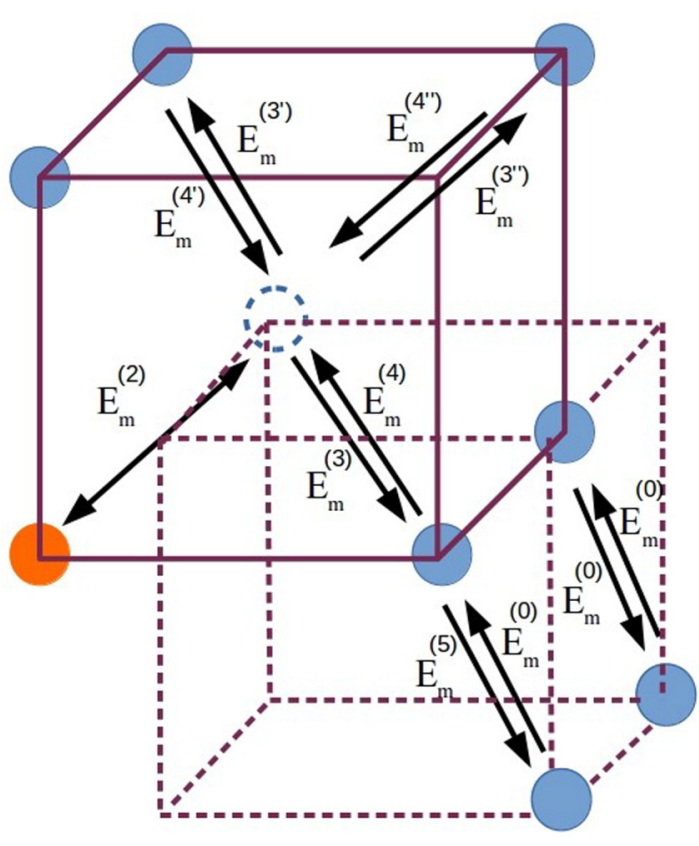

FIG. 2. Distinct migration barriers for different exchanges of the vacancy (dashed circle) in the presence of a Mn atom (orange circle) considering up to $2 n n$ interactions according to Le Claire's 9-frequency model [76]. $E_{m}^{(0)}$ is the migration barrier for any jump that is not affected by the presence of the Mn atom and is equal to the self-diffusion migration barrier of $\mathrm{Fe}$.

are computationally costly, only the migration barriers for the $1 n n$ jumps of the vacancy are considered and are shown in Fig. 2.

In a pure metal, the (solvent) self-diffusion correlation factor $f_{0}$ is a constant for the given crystal structure, being about 0.727 for the bcc crystal symmetry [14]. $f_{2}$ is the solute diffusion correlation factor and is in turn a function of the above mentioned migration frequencies [76]:

$$
\begin{gathered}
f_{2}=\frac{\mu}{2\left(\omega_{2} / \omega_{3}\right)+\mu}, \\
\mu=7-\left(1+0.512\left(\frac{\omega_{0}}{\omega_{4}}\right)\right)^{-1} \\
-2\left(1+1.536\left(\frac{\omega_{0}}{\omega_{4}}\right)\right)^{-1}-\left(1+3.584\left(\frac{\omega_{0}}{\omega_{4}}\right)\right)^{-1} .
\end{gathered}
$$

In the EIM-MC approach, a more extended form of the Le Claire model (nine frequencies) was used. Hence, the expression of the solute diffusion correlation factor $f_{2}$ is

$$
f_{2}=\frac{1+t_{1}}{1-t_{1}}
$$

where $t_{1}$ is expressed as a function of jump frequencies as follows:

$$
t_{1}=-\frac{\omega_{2}}{\omega_{2}+\Gamma_{3}-\frac{\omega_{3} \omega_{4}}{\omega_{4}+F \omega_{5}}-\frac{2 \omega_{3^{\prime}} \omega_{4^{\prime}}}{\omega_{4^{\prime}}+3 F \omega_{0}}-\frac{\omega_{3^{\prime \prime}} \omega_{4^{\prime \prime}}}{\omega_{4^{\prime \prime}}+7 F \omega_{0}}} .
$$

Here $\Gamma_{3}=3 \omega_{3}+3 \omega_{3^{\prime}}+\omega_{3^{\prime \prime}}, \omega_{i}$ are the distinct above mentioned jump frequencies (the corresponding barriers are 
specified in Fig. 2), and $F$ is a structure-dependent parameter, its value is 0.512 in the bec structure [76].

\section{Mixing scheme for the ratio of diffusion coefficients}

The migration barriers in the ferromagnetic state and paramagnetic state yield their respective diffusion ratios, which in turn have a Boltzmann dependence on temperature. A calculation of diffusion ratios at each temperature from first principles is computationally costly, since it involves explicit calculations of migration barriers at these temperatures. Therefore, we use a mixing scheme [77] based on the Ruch model in Eq. (2) to interpolate the migration barriers $E_{m}^{\mathrm{FM}}$, $E_{m}^{\mathrm{PM}}$ of different jumps obtained in the fully ferromagnetic and fully paramagnetic states with

$$
\alpha_{m}=\left(E_{m}^{\mathrm{FM}}-E_{m}^{\mathrm{PM}}\right) / E_{m}^{\mathrm{PM}} .
$$

Here, each vacancy jump has a specific $\alpha_{m}$. The resulting migration barriers are used to calculate the ratio of diffusion coefficients and kinetic correlation factors. For the DFT based SSA relaxation scheme, the absolute diffusion coefficients of $\mathrm{Mn}$ are calculated from the ratio, whereas the self-diffusion coefficients are obtained by the Ruch model [33]. The magnetization values are taken from the experiments of Takeuchi et al. [78]. While the Ruch model is used to interpolate activation energies and migration barriers, the difference between them at an intermediate temperature is calculated as the vacancy formation energy at that temperature.

\section{Explicit temperature dependence of diffusion coefficients}

In order to obtain the explicit temperature dependence of the vacancy formation magnetic free energy, we use our Monte Carlo (MC) approach published previously [61]. At low temperatures, a quantum treatment of spins is necessary for a correct prediction of the magnetic entropy $[31,79,80]$. We therefore adopted the Bose-Einstein statistics in our spinMC simulations [44] up to the Curie point, following the quasi-harmonic approach of Ref. [79].

During these MC simulations, at each $T$, we start performing $5 \times 10^{8}$ spin Metropolis MC steps to reach the equilibrium magnetic state, then 600 spin steps are performed after each atomic MC step, consisting in a $1 n n$ atom-vacancy exchange based on a time residence algorithm. For simplicity, we assume the typical time spent for one atom-vacancy exchange is sufficiently short, so that all the atomic spins are kept frozen while going from the initial to the saddle-point state. However, similar simulations were performed assuming that the spin-variation time is much shorter than the lifetime of both the initial and the saddle-point states, and very close migration barriers were obtained [44].

In Fig. 3 the vacancy formation magnetic free energy in pure $\mathrm{Fe}$ and nearby (1nn) a Mn solute is shown as a function of temperature. While both DFT-SSA and EIM-MC methods are largely in agreement, a systematic difference in the paramagnetic trends can be observed. The factors that could be responsible for this are discussed in Sec. IV. In EIM-MC, in practice, we determine these energies by evaluating the ratio of equilibrium vacancy concentrations of the studied system at a given temperature and at a reference state with a known vacancy formation energy (in our case the perfectly

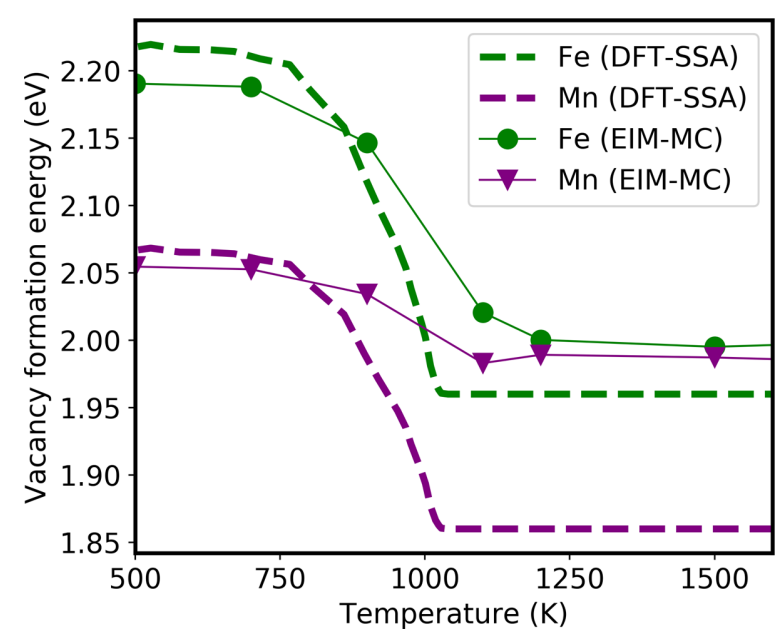

FIG. 3. The vacancy formation energy in pure Fe and at $1 n n$ distance from a Mn solute in Fe, obtained with DFT-SSA and EIM-MC. DFT-SSA values at intermediate temperatures are obtained by subtracting corresponding migration barriers from activation energies, both being extracted from the Ruch model.

$\mathrm{FM}$ bcc $\mathrm{Fe}$ ) via $\mathrm{MC}$ simulations, using the same approach as in Ref. [44].

Using the same MC simulations, we also obtained the magnetic free energy of vacancy migration in the self-diffusion case, and the magnetic free energy barrier for Mn-vacancy and the distinct Fe-vacancy exchanges in the Mn diffusion case. The Fe and Mn diffusion coefficients are obtained by directly simulating the tracer diffusion experiments $[81,82]$. We compute the mean square displacement of the tracers $\left\langle r^{2}\right\rangle$ and the physical time at each temperature [cf. Eq. (6)]. The physical time $t$ is rescaled in order to consider the equilibrium vacancy concentration instead of the actual vacancy concentration of the simulation (the diffusion coefficient is multiplied by the factor $\left.C_{\mathrm{vac}} / C_{\mathrm{MC}}\right)[67,68]$.

\section{Experimental procedure}

Polycrystalline iron rods of $99.98 \mathrm{wt} \%$ purity purchased from HMW-Hauner GmbH \& Co. (Germany) were used. The nominal amounts of selected impurities as indicated by the supplier are reported in Table I.

The cylindrical rods of about $8 \mathrm{~mm}$ diameter were cut by spark erosion into discs of $1.5 \mathrm{~mm}$ in height. The material was annealed at $1143 \mathrm{~K}$ for 10 days below the $\alpha-\gamma$ phase transition temperature in order to reduce the dislocation density. The discs were then mechanically polished to a mirror-like finish. The samples were carefully etched and subjected to a second annealing treatment at $1143 \mathrm{~K}$ for 3 days to remove the mechanical stresses induced by the polishing procedure. The final grain size was measured to be about $500 \mu \mathrm{m}$. All annealing treatments were performed in sealed

TABLE I. Impurity content of the 99.98 wt $\%$ pure Fe samples.

\begin{tabular}{ccccccccr}
\hline \hline Element & $\mathrm{Cu}$ & $\mathrm{Zn}$ & $\mathrm{N}$ & $\mathrm{Si}$ & $\mathrm{S}$ & $\mathrm{C}$ & $\mathrm{P}$ & $\mathrm{O}$ \\
\hline wt. ppm & 900 & 170 & 96 & $<50$ & $<50$ & 20 & $<20$ & 4 \\
\hline \hline
\end{tabular}



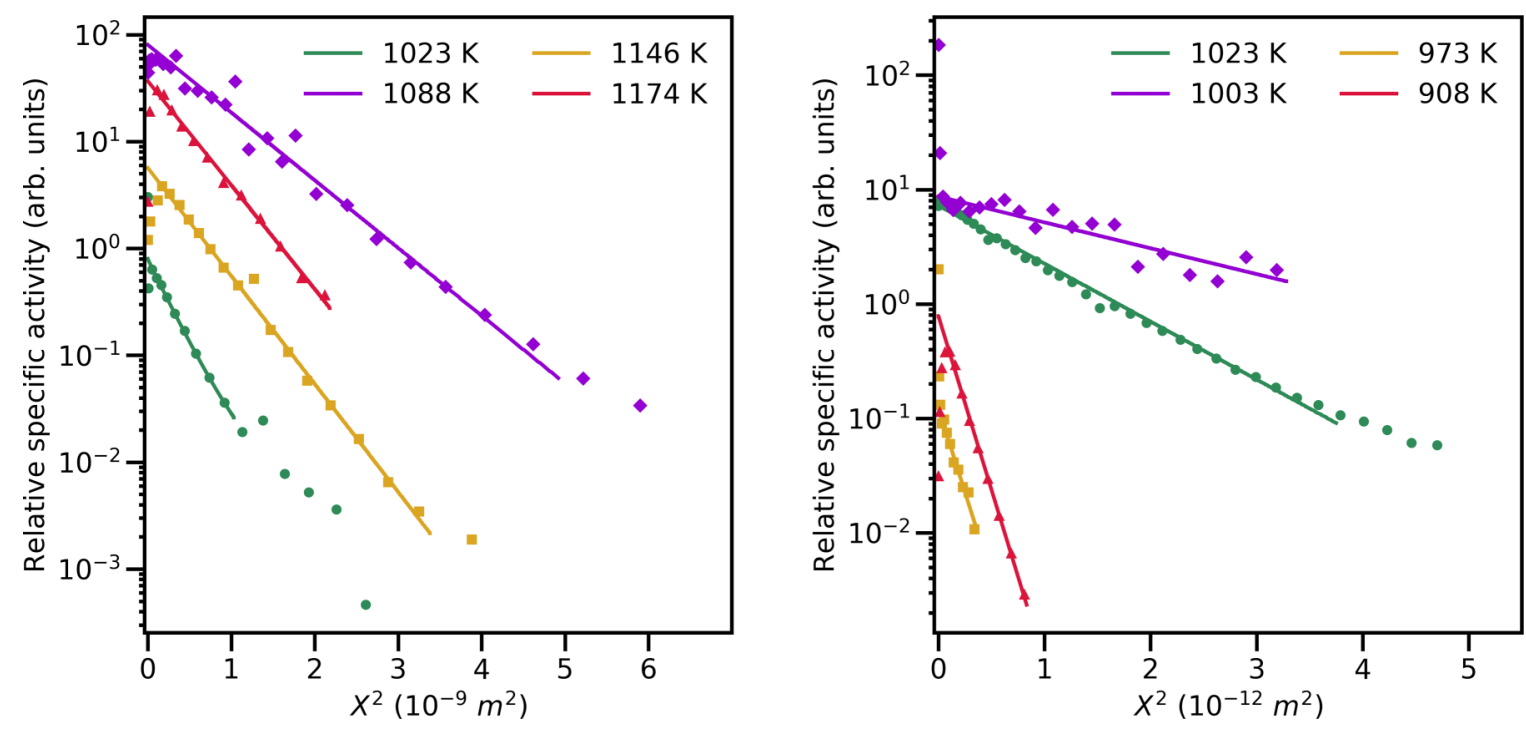

FIG. 4. Penetration profiles for diffusion of ${ }^{54} \mathrm{Mn}$ in bcc Fe obtained by (a) microtome sectioning and (b) ion-beam sputtering. The solid lines represent the fits according to the Gaussian-type solution of the diffusion problem. At larger depths, the measured activities are affected by grain boundary diffusion.

quartz tubes filled with a high-purity argon. The temperature was controlled within $\pm 1 \mathrm{~K}$ with a calibrated $\mathrm{NiCr} / \mathrm{Ni}$ thermocouple. For the annealing treatments the samples were wrapped in carefully cleaned Ta foil to prevent undesirable contamination.

The radiotracer ${ }^{54} \mathrm{Mn}$ (half-time $t_{1 / 2}=312.3 \mathrm{~d}$ ) was purchased as $\mathrm{HCl}$ solution and dissolved in double-distilled water. For diffusion measurements, about $5-7 \mathrm{kBq}$ of the radioactive solution was applied to the sample. Subsequently, the samples were wrapped with a Ta foil, sealed in quartz tubes and subjected to the given diffusion annealing treatments.

The penetration profiles were determined using two sectioning techniques, precision parallel grinding and ion-beam sputtering after higher and lower temperatures of the diffusion annealing treatments (larger and smaller penetration depths), respectively. At 974 and $1033 \mathrm{~K}$, the measurements were performed by both techniques to compare directly the results obtained by different methods and prove the reliability of the data.

In the case of mechanical grinding, the samples were reduced in diameter after annealing (by at least $1 \mathrm{~mm}$ ) to eliminate the lateral and surface diffusion effects. Parallel layers of 3 to $6 \mu \mathrm{m}$ in thickness were successively ground. The thickness was accurately determined by weighing the sample before and after each grinding step on a microbalance with an accuracy $\pm 0.1 \mu \mathrm{g}$.

In the case of ion-beam sputtering, a custom-built device was used [83]. A hole aperture of $3.5 \mathrm{~mm}$ in diameter was used to sputter-off exclusively a central part of the sample. The section thickness was determined assuming a constant sputtering rate (the beam current was controlled to be constant within $\pm 5 \%$ ), weighing the specimen before and after sputtering, and determining the diameter of the sputtering area by optical microscopy.

The section activity of the ${ }^{54} \mathrm{Mn}$ radiotracer was measured using a well-type intrinsic Ge $\gamma$ detector equipped with a $16 \mathrm{~K}$ multichannel energy analyzer; the intensity of the $\gamma$ peak at $834.8 \mathrm{keV}$ was followed.

\section{RESULTS}

\section{A. Experimental diffusion parameters}

Selected penetration profiles measured by the two sectioning techniques are shown in Figs. 4(a) and 4(b). Here, the tracer concentration (which is proportional to the measuredbackground subtracted-relative specific activity divided by the section mass) is plotted against the penetration depth squared $x^{2}$.

The penetration profiles were found to follow either Gauss or complimentary error function solutions depending on the particular initial conditions. At temperatures lower than about $1030 \mathrm{~K}$, an additional short-circuit-type contribution has been observed, Fig. 4, and it was identified as grain boundary diffusion. Since the conditions corresponded to the formal B-type kinetic regime of grain boundary diffusion after Harrison classification [84], the short-circuit contribution was fitted using the Le Clair type of solution [85] as $\exp \left(-q \cdot x^{1.2}\right)$ [86]. Here $q$ is the numerical factor, which determines the corresponding grain boundary diffusivity (the so-called triple product for the given kinetic conditions [86]). This procedure allowed a reliable subtraction of the short-circuit diffusion contribution. In that follows, we will concentrate on the contribution of volume diffusion, is indicated by the solid lines in Figs. 4(a) and $4(b)$.

In Fig. 5, the measured tracer diffusion coefficients are compared with the existing literature data on Mn diffusion in $\alpha$-Fe. In the paramagnetic state, the present results agree nicely with the data of Nohara et al. [38] and Lübbehusen [15], while larger deviations are obvious in the ferromagnetic state. In the present paper, Mn diffusion is for the first time measured down to low temperatures using the ion-beam sputtering technique (circles), providing reliable estimates of the diffusion retardation induced by the magnetic transition. 


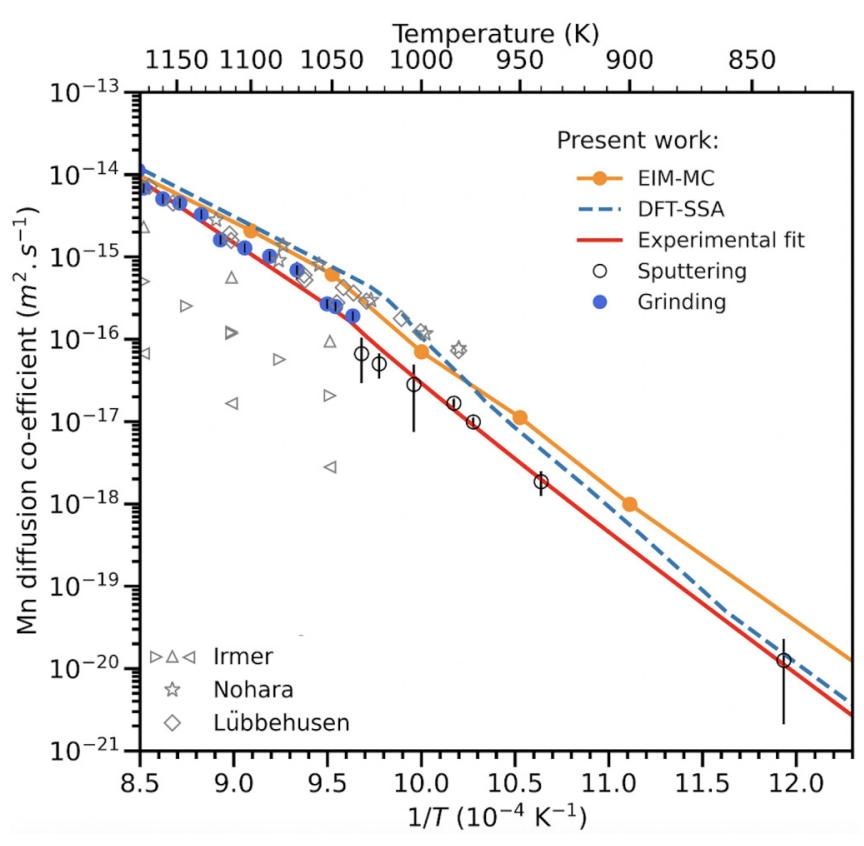

FIG. 5. Diffusion coefficients of Mn in bcc Fe as function of the inverse temperature, obtained from DFT-SSA, EIM-MC and experimental measurements (circles). At higher temperatures, the determined experimental uncertainties are typically less than the symbol size. The published results of Nohara et al. [38] (stars), Lübbehusen [15] (diamonds), and Irmer et al. [40] (triangles) are shown for comparison.

The results of Irmer et al. [40] measured in paramagnetic Fe deviate strongly from all existing datasets that might be explained by a relatively low purity of the iron samples used in Ref. [40]. (The different triangle types refer to different sorts of pure iron, for further details see the corresponding paper.)

The present data measured for the first time in an extended temperature interval substantiate unambiguously a certain impact of the magnetic transition on Mn diffusion in pure $\alpha-\mathrm{Fe}$, Fig. 5, filled symbols. In fact, diffusion rates of Mn are slower in the ferromagnetic region comparing to a simple Arrheniustype extrapolation from the paramagnetic area.

The actual values are listed together with the time of the diffusion annealing treatments in Table II. The experimental uncertainties of the measured tracer diffusion coefficients are indicated as upper and lower bounds of the values, which could still be determined using different reasonable fitting procedures. The standard uncertainties of the diffusion coefficients can be estimated to be smaller than $\pm 20 \%$.

A moderate impact of the magnetic transition at the Curie temperature $T_{\mathrm{C}}$ on the rate of $\mathrm{Mn}$ diffusion in bcc-Fe is seen. For a more precise evaluation, the parameter $\alpha$ is determined after Ruch, Eq. (2), in an Arrhenius plot of the diffusion constant $\ln \left(D(T) / D_{0}^{\mathrm{PM}}\right)$ as a function of the reduced magnetization squared, provided the parameters of Mn diffusion in the paramagnetic state are known. The latter are determined as: $D_{0}^{\mathrm{PM}}=1.35_{-1.1}^{+4.8} \cdot 10^{-1} \mathrm{~m}^{2} / \mathrm{s}, E_{a}^{\mathrm{PM}}=297 \pm 14 \mathrm{~kJ} / \mathrm{mol}$, the resulting value of $\alpha=0.056 \pm 0.024$.
TABLE II. Temperature $T$, time $t$, and the determined diffusion coefficients $D$ for $\mathrm{Mn}$ volume diffusion in bcc-Fe. The sectioning method ( $\mathrm{M}=$ mechanical grinding; $\mathrm{S}=$ ion-beam sputtering) and the type of solution used $(\mathrm{G}=$ Gauss; $\mathrm{E}=$ complementary error function) are indicated.

\begin{tabular}{|c|c|c|c|c|}
\hline $\begin{array}{l}T \\
(\mathrm{~K})\end{array}$ & $\begin{array}{c}t \\
\left(10^{3} \mathrm{~s}\right)\end{array}$ & $\begin{array}{c}D \\
\left(\mathrm{~m}^{2} / \mathrm{s}\right)\end{array}$ & Method & Solution \\
\hline 1178 & 14.4 & $1.14_{-0.42}^{+0.08} \cdot 10^{-14}$ & M & G \\
\hline 1174 & 16.2 & $6.89_{-0.79}^{+0.24} \cdot 10^{-15}$ & M & $\mathrm{G}$ \\
\hline 1160 & 28.8 & $5.08_{-0.34}^{+0.55} \cdot 10^{-15}$ & M & G \\
\hline 1148 & 23.4 & $4.53_{-0.05}^{+0.05} \cdot 10^{-15}$ & M & G \\
\hline 1133 & 86.4 & $3.29_{-0.53}^{+0.27} \cdot 10^{-15}$ & M & G \\
\hline 1120 & 55.8 & $1.62_{-0.10}^{+0.12} \cdot 10^{-15}$ & M & G \\
\hline 1104 & 167.4 & $1.29_{-0.12}^{+0.02} \cdot 10^{-15}$ & M & $\mathrm{E}$ \\
\hline 1088 & 171 & $1.02_{-0.15}^{+0.08} \cdot 10^{-15}$ & M & G \\
\hline 1071 & 244.8 & $6.95_{-0.19}^{+1.75} \cdot 10^{-16}$ & M & G \\
\hline 1053 & 252 & $2.70_{-0.01}^{+0.05} \cdot 10^{-16}$ & M & G \\
\hline 1048 & 259.2 & $2.51_{-0.20}^{+0.37} \cdot 10^{-16}$ & M & G \\
\hline 1038 & 432 & $1.93_{-0.06}^{+0.06} \cdot 10^{-16}$ & M & G \\
\hline 1033 & 3.6 & $6.69_{-0.16}^{+0.38} \cdot 10^{-17}$ & S & G \\
\hline 1032 & 518.4 & $1.34_{-0.11}^{+0.01} \cdot 10^{-16}$ & M & G \\
\hline 1028 & 691.2 & $1.10_{-0.39}^{+0.47} \cdot 10^{-16}$ & M & G \\
\hline 1023 & 3.6 & $5.04_{-0.07}^{+1.70} \cdot 10^{-17}$ & $S$ & $\mathrm{E}$ \\
\hline 1022 & 1188 & $5.54_{-0.31}^{+0.03} \cdot 10^{-17}$ & M & $\mathrm{E}$ \\
\hline 1004 & 14.4 & $2.84_{-0.59}^{+3.09} \cdot 10^{-17}$ & S & G \\
\hline 983 & 16.2 & $1.68_{-0.64}^{+0.17} \cdot 10^{-17}$ & S & G \\
\hline 973 & 5097.6 & $8.87_{-2.00}^{+1.17} \cdot 10^{-18}$ & M & G \\
\hline 973 & 3.6 & $9.95_{-4.28}^{+1.13} \cdot 10^{-18}$ & S & G \\
\hline 940 & 3.6 & $1.87_{-1.00}^{+0.63} \cdot 10^{-18}$ & S & G \\
\hline 838 & 3024 & $1.26_{-0.43}^{+1.95} \cdot 10^{-20}$ & S & $\mathrm{G}$ \\
\hline
\end{tabular}

\section{B. Computed diffusion parameters}

DFT calculations for bcc Fe yield in the ferromagnetic limit a vacancy formation energy of $2.22 \mathrm{eV}$. Employing the SSA relaxation technique, one observes that the energy is reduced to $1.96 \mathrm{eV}$ in the paramagnetic limit [32]. Important for the present evaluation is the first nearest-neighbor binding energy determined according to Eq. (3), which reveals an attractive interaction between the $\mathrm{Mn}$ and the vacancy. Its value is slightly larger in the ferromagnetic state $(0.15 \mathrm{eV})$ as compared to the paramagnetic state $(0.10 \mathrm{eV})$. Combining both results, the difference between the vacancy formation energies of $2.07 \mathrm{eV}$ and $1.86 \mathrm{eV}$, respectively in the ferromagnetic and paramagnetic states, is therefore slightly reduced in the presence of Mn. The second nearest-neighbor Mn-vacancy interaction is weak for both ferromagnetic and paramagnetic states, as the corresponding binding energies are $0.06 \mathrm{eV}$ and $0.03 \mathrm{eV}$.

The DFT results for the migration energies for the nearestneighbor jumps in the two magnetic states are reported in Table III. It is to be noted that the migration barriers in the paramagnetic state are considerably lower as compared to their ferromagnetic counterparts. This is to a large extent due to the local relaxation effects in the paramagnetic state, which 
TABLE III. The migration energies for the nearest-neighbor jumps of the vacancy in both ferromagnetic and paramagnetic states obtained with DFT-SSA and EIM-MC. In the DFT case, the barriers for $2 n n$ exchanges are assumed to be equal to $E_{m}^{(0)}$. Values from previous DFT papers (available only for the ferromagnetic case) are shown for comparison.

\begin{tabular}{|c|c|c|c|c|c|}
\hline \multicolumn{6}{|c|}{ Migration energies (eV) } \\
\hline & \multicolumn{3}{|c|}{ Ferromagnetic } & \multicolumn{2}{|c|}{ Paramagnetic } \\
\hline & DFT-FM & EIM-MC & other DFT & DFT-SSA & EIM-MC \\
\hline$E_{m}^{(0)}$ & 0.73 & 0.71 & $0.70[41], 0.70[42]$ & 0.43 & 0.41 \\
\hline$E_{m}^{(2)}$ & 0.46 & 0.41 & $0.42[41], 0.41[42]$ & 0.41 & 0.31 \\
\hline$E_{m}^{(3)}$ & 0.64 & 0.69 & $0.66[41], 0.64[42]$ & 0.42 & 0.39 \\
\hline$E_{m}^{(4)}$ & 0.58 & 0.63 & $0.61[41], 0.60[42]$ & 0.36 & 0.37 \\
\hline$E_{m}^{\left(3^{\prime}\right)}$ & & 0.70 & $0.70[41], 0.68$ [42] & & 0.37 \\
\hline$E_{m}^{\left(4^{\prime}\right)}$ & & 0.59 & $0.55[41], 0.58[42]$ & & 0.37 \\
\hline$E_{m}^{\left(3^{\prime \prime}\right)}$ & & 0.71 & $0.66[41], 0.66[42]$ & & 0.42 \\
\hline$E_{m}^{\left(4^{\prime \prime}\right)}$ & & 0.60 & $0.55[41], 0.56[42]$ & & 0.39 \\
\hline$E_{m}^{(5)}$ & & 0.72 & $0.76[41]$ & & 0.41 \\
\hline
\end{tabular}

are included within the SSA approach along with the effects due to disorder in magnetic configuration. Furthermore, the migration barrier for the jump that associates the $\mathrm{Mn}$ atom and the vacancy $\left(E_{m}^{(4)}\right)$ is lower than the migration barrier for the jump that dissociates $\left(E_{m}^{(3)}\right)$ them, for both the magnetic states. This observation can be related to the attractive interaction between the $\mathrm{Mn}$ atom and the vacancy. It is also remarkable that the Mn diffusion barrier is similar to the self-diffusion migration barrier in the paramagnetic state $(0.1 \mathrm{eV}$ higher $)$, while it is substantially smaller $(0.28 \mathrm{eV})$ than the self-diffusion value in the ferromagnetic state.

The resulting activation energies, defined as the sum of vacancy formation energy and migration energy in pure bcc-Fe, are $2.95 \mathrm{eV}$ and $2.39 \mathrm{eV}$ in the ferromagnetic and paramagnetic states. In the vicinity of single $\mathrm{Mn}$ atoms in bcc Fe, the values are $2.53 \mathrm{eV}$ and $2.27 \mathrm{eV}$, respectively. Therefore, the difference in activation energies of the two magnetic states decrease considerably on the introduction of Mn solute atom. This reduces the kink in the diffusion-coefficients vs temperature curve.

For EIM-MC results, in order to obtain the temperature dependence of the migration barriers and in particular, of the barrier for the Mn jump into the vacancy $E_{m}^{(2)}$, migration simulations for the exchange frequencies $\omega_{i}$ are employed. Using the residence time algorithm, the simulation contains $10^{9}$ atomic-MC steps. Between these atomic jumps, 100 spinMC steps are performed among the whole system, and 500 more are performed among the five nearest-neighbor shells of the vacancy. The average $E_{m}^{(2)}$ barrier obtained is shown in Fig. 6 (symbols) and in Table III. These results are compared with the DFT for the paramagnetic state (dashed lines), which is based on the SSA relaxation technique.

The migration barriers obtained with the two methods overall show the same trends and are in good agreement for both the magnetic states. The ferromagnetic migration barriers obtained by previous DFT papers [41,42] are in good agreement with our study as shown in Table III. The different migration barriers in the paramagnetic state are not reported in

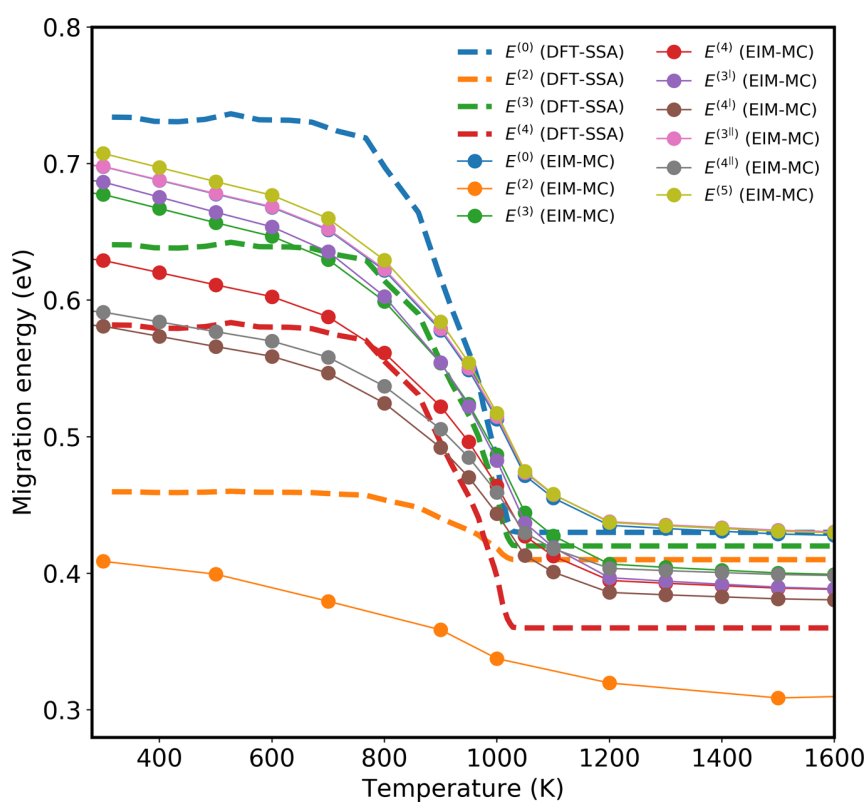

FIG. 6. The vacancy migration energies obtained by considering up to $2 \mathrm{nn}$ interactions from EIM and $1 \mathrm{nn}$ interactions from interpolated DFT results according to the Ruch model.

the literature before. We also note here that the ferromagnetic Mn-vacancy binding energy obtained in our study $(0.15 \mathrm{eV}$ for both the methods) is in good agreement with the literature values $(0.15 \mathrm{eV}$ [42], $0.17 \mathrm{eV}$ [41]). Among different barriers in the paramagnetic state, we note that the Mn barrier has the largest deviation between these two methods (of about $0.1 \mathrm{eV}$ ). The EIM-MC trends for the Mn-diffusion barrier in comparison to the self-diffusion barrier are similar as in the DFT-SSA calculations, with the only difference that it is now lower by $0.1 \mathrm{eV}$ in the paramagnetic state for the EIM-MC case. On the other hand, the difference is only $0.02 \mathrm{eV}$ in the case of DFT-SSA. In the paramagnetic limit, another interesting observation is that $E_{m}^{(4)}$ is the lowest barrier for the DFT-SSA results, whereas $E_{m}^{(2)}$ (or the Mn barrier) is the lowest for EIM-MC. Nonetheless, for both the methods, different migration barriers show a much smaller scatter (or exhibit similar values) in the paramagnetic case as compared to the ferromagnetic limit.

We compare the temperature dependence of the Mn diffusion coefficient obtained by the experiment, with those obtained by DFT-SSA and EIM-MC in Fig. 5. It can be noticed that in the case of $\mathrm{Mn}$ diffusion, the deviation from Arrhenius law near $T_{C}$ is small and similar for experiments, EIM-MC model and DFT-SSA. More precisely, the difference between the paramagnetic and the ferromagnetic activation magnetic free energy of Mn diffusion is reduced in comparison to the self-diffusion counterpart.

\section{DISCUSSION}

\section{A. Difference between Mn diffusion and Fe self-diffusion}

The main observation of Fig. 5 is that the Mn diffusion curve is much more linear (on a logarithmic scale) than the self-diffusion curve, which exhibits a significant change of 


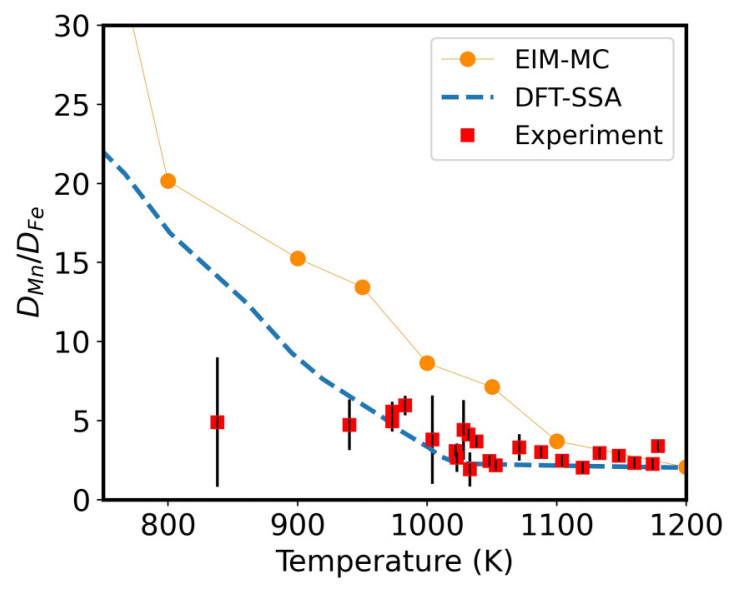

FIG. 7. Ratio between the Mn diffusion coefficient and the Fe self-diffusion coefficient.

slope between the ferromagnetic and paramagnetic regimes. The nature of a potential "kink" at the Curie temperature can probably be considered to be most systematically explored within the EIM-MC approach, since it has direct access to a large temperature range including $T_{C}$. However, as the EIM-MC is not free of approximations, the comparison with the DFT-SSA method is essential.

In fact, the two approaches differ in some essential aspects, representing different limits:

(1) In the DFT-SSA case, it is assumed that the time-scale of fluctuations in the magnetic degrees of freedom is much shorter than the migration time of the vacancy, while all the atomic spins are kept frozen in the MC simulations when going from the initial to the saddle-point state.

(2) The DFT based SSA relaxations are different from the EIM, where the paramagnetic state is modelled based on DFT calculations with the geometry of the ferromagnetic state.

(3) The DFT based SSA relaxations neglict Mn-vacancy interactions beyond $1 n n$ distance because of the high computational cost. Up to $2 n n$ interactions are considered for the parametrization of the EIM, while only 1 nn jumps are considered for the MC simulations.

(4) The SSA relaxations are performed with a larger lattice constant to include the effect of thermal expansion.

(5) The EIM-MC explicitly determines diffusion coefficients at intermediate temperatures while the SSA relaxations assume a fully paramagnetic state and an interpolation scheme is used for intermediate temperatures.

As a consequence, the two theoretical methods show noticeable quantitative differences for some parameters (for example, a systematic difference in predicting vacancy formation energies). Nevertheless, for the essential results of this study, namely the diffusion coefficients as shown in Fig. 5 they are largely in good agreement with each other and the experiments, supporting their predictive power.

This further strengthens the reliability of the ratio between the Mn diffusion coefficient and the Fe self-diffusion coefficient as analyzed in Fig. 7 for both the theoretical methods and the experiments. The lowest temperature for which results are available from the experimental side is $830 \mathrm{~K}$, at which the ratio is about 5 . The ratio decreases with temperature up to $T_{C}$

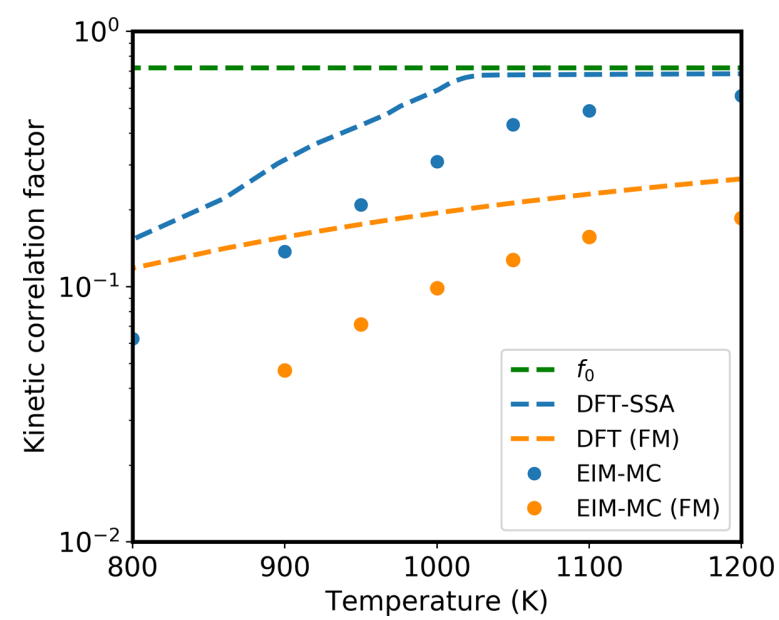

FIG. 8. Kinetic correlation factors of Fe self-diffusion $\left(f_{0}\right)$ and Mn diffusion $\left(f_{2}\right)$. The blue dashed line and blue dots represent $f_{2}$ obtained by DFT-SSA and EIM-MC, respectively. The $f_{2}$ kinetic correlation factor for the pure FM state is displayed to highlight the impact of magnetic disorder and is represented by an orange dashed line for DFT-SSA and orange dots for EIM-MC.

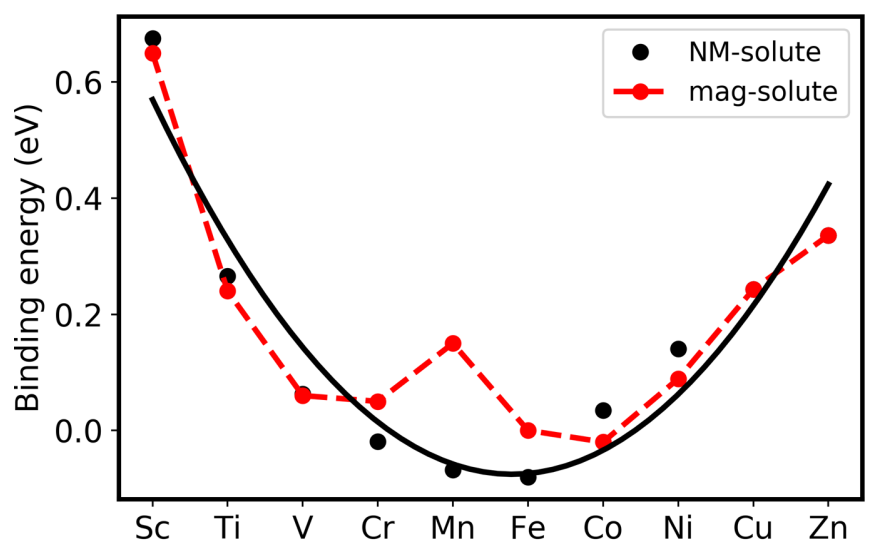

(a)

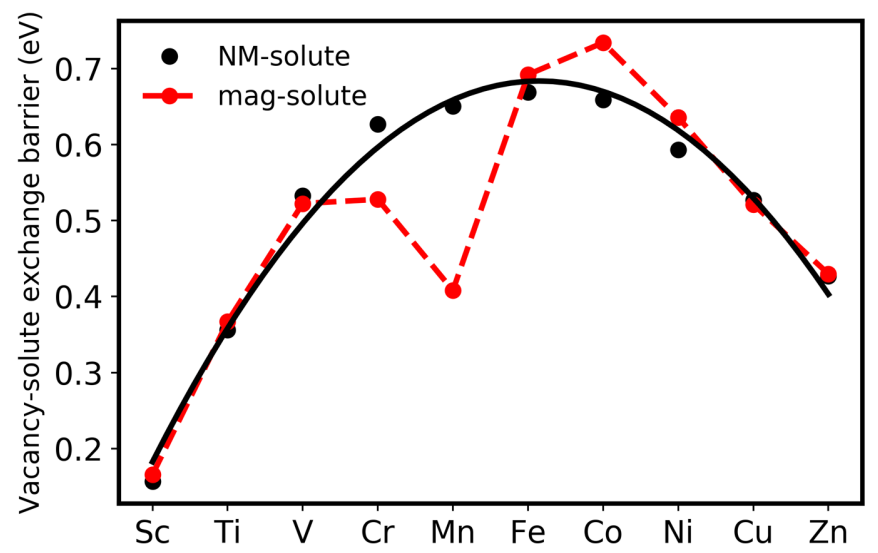

(b)

FIG. 9. (a) Solute-vacancy binding energy and (b) exchange barrier in FM bcc Fe from DFT. We show the values with fully relaxed magnetic moments (magnetic ground state) and with the solute moment constrained to zero. Data for the latter case are fitted and represented by black solid lines. 


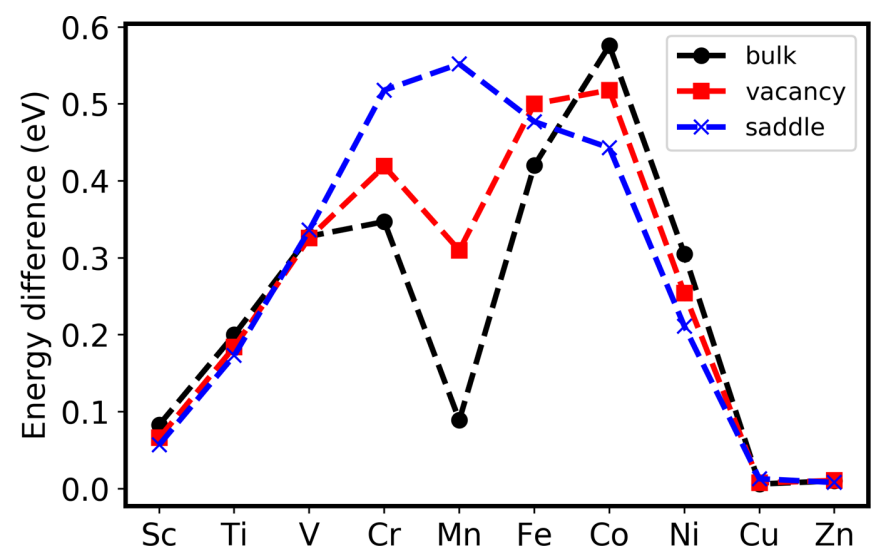

(a)

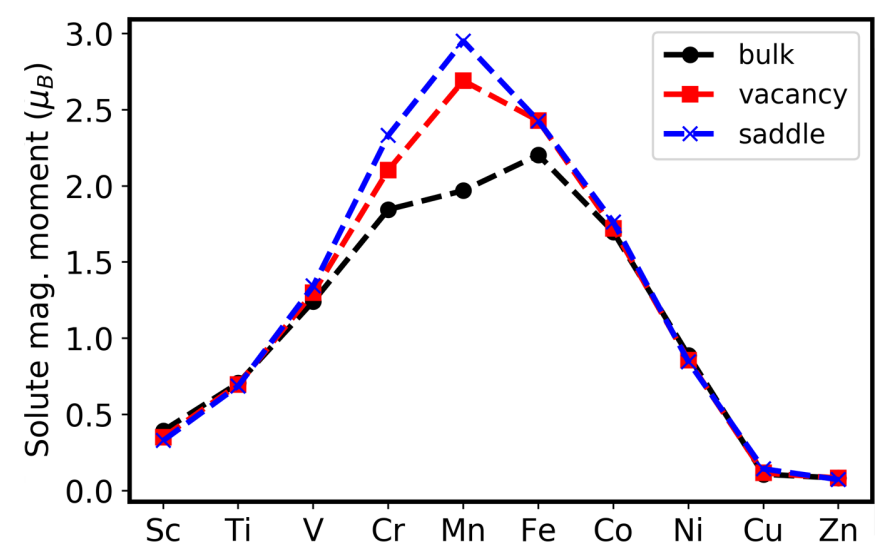

(b)

FIG. 10. (a) Energy difference between the cases with the solutemoment relaxed (magnetic ground state) and constrained to zero. (b) Relaxed magnetic moment magnitude of the solute (in $\mu_{B}$ ). Data are shown for the solute in the bcc Fe bulk, next to a vacancy, or in the saddle-point position.

where its value is between 2 and 3 . Beyond $T_{C}$, this ratio has at about $1178 \mathrm{~K}$ (which is the highest available data) a value between 2 and 4 . The decrease is much more pronounced in the theoretical investigations. In fact, the calculated ratio for both DFT-SSA and EIM-MC is about 20 at $800 \mathrm{~K}$. It decreases and reaches about 2 for DFT-SSA and about 7 for the EIM-MC at $T_{C}$, getting again close to the experimental results. Hence, the theoretical investigations consistently indicate that the ferromagnetic regime explains the less pronounced kink for the Mn diffusion coefficient as compared to the self-diffusion behavior.

To analyze this effect further, we show the kinetic correlation factors for Mn diffusion in Fig. 8. It increases with temperature up to an asymptotic limit of 0.73 , which is the $f_{0}$ value in pure bcc iron. Again, both EIM and DFT are in good agreement. To clarify the role of magnetic disorder on the $f_{2}$, we have performed similar MC simulations for $\mathrm{Mn}$ diffusion, but imposing a perfect FM order for all atomic-MC temperatures. The results show that the kinetic correlation factor of Mn diffusion increases more slowly when the magnetic disorder is absent. The same is true for DFT results where only ferromagnetic migration barriers are used to determine the kinetic correlation factor.

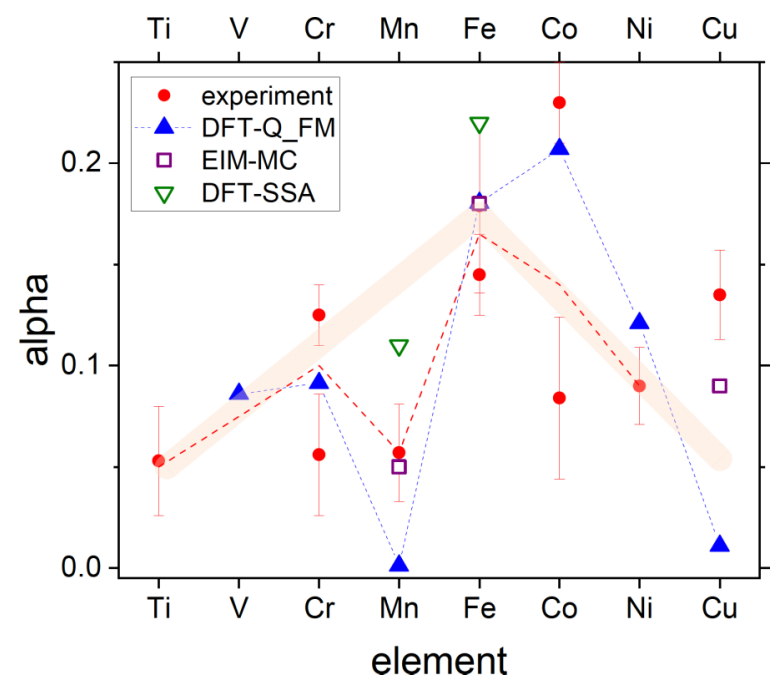

FIG. 11. A comparison of $\alpha$ values in the Ruch model, Eq. (2), determined using the published experimental diffusion coefficients (red filled circles, Table IV), or DFT-based calculations. The experimental trend (red dashed line) is determined by averaging the data published for the same solute. The thick line indicates a hypothetical trend for $\alpha$ as the function of element number for the $3 d$ transition metal elements.

The more linear trend of diffusion coefficients (in logarithmic scale) can be understood from the analysis of binding energies and migration barriers in the ferromagnetic and paramagnetic states. In the ferromagnetic state, both DFT and EIM-MC show a stronger Mn-vacancy binding energy (DFT $\sim 0.15 \mathrm{eV}$, EIM-MC $\sim 0.15 \mathrm{eV}$ ), compared to the weaker binding energy in the paramagnetic state (DFT-SSA $\sim 0.10 \mathrm{eV}$, EIM-MC $\sim 0.02 \mathrm{eV}$ ). In addition, the migration barrier of Mn solute diffusing in the paramagnetic state of $\mathrm{Fe}$ differs only slightly from the migration barrier of $\mathrm{Fe}$ in pure $\mathrm{Fe}$, while the difference is larger in the ferromagnetic state. Also, the migration energies for different exchanges of a vacancy at $1 n n$ distance of a $\mathrm{Mn}$ atom in Fe host are closer to the $\mathrm{Fe}$ migration energy in pure $\mathrm{Fe}$ in the paramagnetic regime, compared to the ferromagnetic regime (Table III, Fig. 6). Together with the effect of magnetic disorder on the kinetic correlation factor $f_{2}$ (Fig. 8), this observation confirms the dominance of magnetic disorder over chemical interaction effects in this very dilute Fe-Mn system at high temperatures.

\section{B. Anomalous behavior of Mn}

As can be confirmed by further simulations for $\mathrm{Cu}$ solutes [44], it seems to be a general phenomenon of solutes in bcc iron that they experience a similar activation magnetic free energy $\left(E_{a}\right)$ in the paramagnetic regime, while their $E_{a}$ in the ferromagnetic state can be significantly different. Therefore, the size of the "kink" and the $\Delta E_{a}$ of different solutes are mainly dictated by the $E_{a}$ in the ferromagnetic state. To better understand this, we have further extended the study of solute-vacancy binding energy and the solute-vacancy exchange barrier in FM $\alpha$-Fe performed for Mn above to all the $3 d$ elements. 
TABLE IV. Best fits according to the Ruch model [33] of published experimental data using the magnetization data from Ref. [89].

\begin{tabular}{lcccc}
\hline \hline & Ref. & $\begin{array}{c}D_{0}^{\mathrm{PM}} \\
\left(\mathrm{m}^{2} / \mathrm{s}\right)\end{array}$ & $\begin{array}{c}E_{a}^{\mathrm{PM}} \\
(\mathrm{eV} / \mathrm{at})\end{array}$ \\
\hline $\mathrm{Ti}$ & {$[90]$} & $1.35_{-1.1}^{+5.4}$ & $3.22 \pm 0.15$ & $\alpha$ \\
$\mathrm{Cr}$ & {$[91]$} & $3.74_{-2.8}^{+11} \times 10^{-3}$ & $2.83 \pm 0.13$ & $0.053 \pm 0.027$ \\
& {$[36]$} & $1.48_{-0.8}^{+2.0} \times 10^{-2}$ & $2.91 \pm 0.08$ & $0.070 \pm 0.03$ \\
$\mathrm{Mn}$ & present paper & $1.35_{-1.1}^{+4.8} \times 10^{-1}$ & $3.08 \pm 0.14$ & $0.125 \pm 0.015$ \\
$\mathrm{Fe}$ & {$[4]$} & $8.85_{-8.3}^{+146} \times 10^{-4}$ & $2.70 \pm 0.27$ & $0.056 \pm 0.024$ \\
& {$[4]$} & $6.44_{-5.5}^{+36} \times 10^{-5}$ & $2.47 \pm 0.17$ & $0.145 \pm 0.058$ \\
$\mathrm{Co}$ & {$[92]$} & $2.76_{-2.8}^{+56} \times 10^{-4}$ & $2.60 \pm 0.29$ & $0.23 \pm 0.04$ \\
& {$[93]$} & $1.48_{-1.42}^{+36} \times 10^{-1}$ & $3.26 \pm 0.3$ & $0.084 \pm 0.05$ \\
$\mathrm{Ni}$ & {$[35]$} & $8.28_{-5.4}^{+13} \times 10^{-3}$ & $2.90 \pm 0.08$ & $0.090 \pm 0.02$ \\
$\mathrm{Cu}$ & {$[13]$} & $4.94_{-3.0}^{+7.8} \times 10^{-3}$ & $2.77 \pm 0.09$ & $0.13 \pm 0.03$ \\
\hline \hline
\end{tabular}

It is well known that, divers properties of the $3 d$ magnetic elements ( $\mathrm{Cr}, \mathrm{Mn}, \mathrm{Fe}, \mathrm{Co}, \mathrm{Ni})$ show a deviation from the expected parabolic behavior as a function of the $d$ band filling $[87,88]$. We have therefore computed the binding energy in Fig. 9(a) and the exchange energy barrier in Fig. 9(b) under two different conditions: either, imposing the solute moment to zero while relaxing the moment of all other Fe atoms, or relaxing the magnetic moment of all the atoms. In the former case, we observe the expected nearly-parabolic behavior, while we note deviation from the overall trend for some of the magnetic elements, especially in the case of Mn. For Mn, the very strong deviation leads to an increase of vacancyMn binding energy and a decrease of vacancy-Mn exchange barrier, both of around $0.2 \mathrm{eV}$. Such a decrease of the activation energy of around $0.4 \mathrm{eV}$ in the ferromagnetic regime, makes it approaching the value of $E_{a}$ in the paramagnetic state. Therefore, the "kink" is significantly reduced for Mn diffusion in $\alpha$-iron, as shown both experimentally and by DFT-based simulations.

These deviations can be rationalized by comparing the energy differences between the system with and without imposing the solute moment to zero, with the three local environments for the solute. We see from Fig. 10(a) that, at the saddle point position, they follow the solute moment magnitude [Fig. 10(b)]. Please note that Fig. 10(b) only shows the spin magnitudes. For more details and in agreement with previous calculations [88], the solutes with a smaller (respec- tively larger) number of $3 d$ electrons than that of Fe show an antiparallel (respectively parallel) spin to the Fe moments, in the magnetic ground state. In the bulk and next to the vacancy, however, Mn (and Co to a less extend) deviate from the trend that the energy difference follows the solute moment magnitude.

For Mn, the deviations are due to the strong localenvironment dependence of these energy differences shown in Fig. 10(b), with the smallest $\Delta E$ for a Mn solute in the bulk, and then next to the vacancy. As a specificity of Mn, DFT calculations predicted the presence of two energy minima for a Mn solute in bcc iron [27,28]. The antiferromagnetic-Mn (with a large Mn moment antiparallel to Fe moments) being the ground state, and the ferromagnetic-Mn state, showing a small Mn moment, parallel to the Fe moments. The Mn $\Delta E \mathrm{~s}$ in Fig. 10(a), follow the same trend of the energy difference between the antiferromagnetic-Mn and the ferromagnetic-Mn states. In bulk, they have only $0.07 \mathrm{eV}$ of energy difference. This ferromagnetic-Mn state is energetically less favorable next to a vacancy [with a $\Delta E$ (ferromagnetic-Mn antiferromagnetic-Mn) $=0.28 \mathrm{eV}$, and this $\Delta E$ is even larger for a $\mathrm{Mn}$ at the saddle-point position $(0.53 \mathrm{eV})$.

We believe that this anomaly of $\mathrm{Mn}$ is also reflected in the $\alpha$ values according to the Ruch model in Eq. (2). We already identified a different behavior of the "kink" reflected by the $\alpha$ value for Mn solute diffusion and Fe self diffusion. The situation is now more systematically analyzed in Fig. 11. Here,

TABLE V. EIM parameters: Magnetic and chemical interaction parameters between two atoms, depending on their relative distance, respective species, and the eventual proximity of a vacancy (in meV).

\begin{tabular}{|c|c|c|c|c|c|}
\hline$J_{F e-F e}$ & -3.39 & -2.26 & -0.83 & 0.42 & 0.44 \\
\hline$J_{M n-M n}$ & 1.51 & 1.30 & 0.26 & -0.98 & 0.53 \\
\hline$V_{\mathrm{Fe}-\mathrm{Fe}}$ & -10.85 & 8.18 & 0 & 0 & 0 \\
\hline$V_{M n-M n}$ & 4.63 & -1.93 & -1.06 & -0.19 & 0.25 \\
\hline$V_{F e-M n}$ & -6.09 & 3.75 & 0 & 0 & 0 \\
\hline$V_{F e-M n}(1 n n v a c)$ & 30.2 & -17.3 & 0 & 0 & 0 \\
\hline$J_{0}(2 n n v a c)$ & 0.189 & 0.212 & 0.151 & 0.267 & 0.110 \\
\hline$V_{F e-M n}(2 n n v a c)$ & -30.2 & 17.3 & 0 & 0 & 0 \\
\hline
\end{tabular}


TABLE VI. EIM parameters: Magnetic on-site terms of Fe and Mn atoms, depending on the presence or not of a vacancy (in meV). Star superscript means saddle-point. The superscripts 0,1 , and 2 in this table refer respectively to the absence of vacancy in the two nearest-neighbor shells of the concerned atom (0), the presence of a vacancy in its first neighbor shell (1) and the presence of a vacancy in its second neighbor shell (2).

\begin{tabular}{lcccccrrr}
\hline \hline Interactions & $A^{(0)}$ & $B^{(0)}$ & $A^{(1)}$ & $B^{(1)}$ & $A^{(2)}$ & $B^{(2)}$ & $A^{*}$ \\
\hline $\mathrm{Fe}$ & -259.0 & 27.6 & -250.2 & 24.7 & -285.7 & 35.2 & -170.1 \\
$\mathrm{Mn}$ & -37.70 & 6.93 & -116.2 & 8.54 & -37.70 & 6.93 & -116.2 & 8.54 \\
\hline \hline
\end{tabular}

the experimental data are based on Table IV, while the DFT data are obtained by calculating the element specific activation energy $\left(E_{a}\right)$ only in the ferromagnetic state and assuming, as a first approximation, that the paramagnetic $E_{a}$ value is identical to that of $\mathrm{Fe}$. We can observe a systematic trend of the $\alpha$ value with the filling of the $3 d$ shell both in experiment as well as in DFT calculations. Mn clearly deviates from this trend in the sense that it does not follow the linear increase with $3 d$ filling from Ti up to Fe. Instead, Mn shows an $\alpha$ value that is comparable to that of $\mathrm{Ti}$ and $\mathrm{Cu}$, which are largely unaffected by the magnetic environment.

\section{CONCLUSIONS}

In summary, we successfully employed tracer diffusion experiments, DFT based SSA relaxations, and on-lattice Monte Carlo simulations using effective interaction models parameterized on DFT data to efficiently predict atomic diffusion properties of $\mathrm{Mn}$ in pure $\alpha$-Fe accross the magnetic transition temperature. The two theoretical approaches compliment each other and arrive at similar physical conclusions, thereby strengthening the reliability of the results. For the first time, the temperature dependence of $\mathrm{Mn}$ tracer diffusion in $\alpha-\mathrm{Fe}$ is measured down to relatively low temperatures allowing a reliable determination of the impact of the magnetic transition on the diffusion behavior.

We predict a reduced "kink" in the diffusion-coefficient temperature evolution for $\mathrm{Mn}$, compared to the case of $\mathrm{Fe}$ and other $3 d$ solutes. This result is in excellent agreement with the experimental data, and can be explained by the smaller difference between the ferromagnetic and paramagnetic activation enthalpies in the Mn case compared to self-diffusion of Fe. As the paramagnetic activation enthalpies are rather similar for all the elements, i.e., Ti [90], $\mathrm{Cu}$ [10-13], Mn (present paper), Fe $[2,3,5,8]$, Co $[35,92,93]$, this atypical behavior of $\mathrm{Mn}$ is dictated by the atypical value of the activation enthalpy in the low-temperature ferromagnetic regime. Indeed, as predicted by previous DFT studies [87], this value for Mn presents the strongest deviation from the standard parabolic trend, due to the half-filled $3 d$ band of $\mathrm{Mn}$, inducing the presence of two magnetic minima for a $\mathrm{Mn}$ atom in bec-iron.

TABLE VII. EIM parameters. Parameters of the polynomial function ensuring the local Mn concentration dependence of Fe-Mn magnetic interaction parameters (in meV)

\begin{tabular}{lccccc}
\hline \hline Interaction & $a$ & $b$ & $c$ & $d$ & $e$ \\
\hline Value (meV) & $6.50 \mathrm{E}-8$ & $-8.40 \mathrm{E}-6$ & $3.83 \mathrm{E}-4$ & $-7.16 \mathrm{E}-3$ & $3.04 \mathrm{E}-2$ \\
\hline \hline
\end{tabular}

We find that chemical-interaction effects due to the presence of the solute are significantly erased by the magnetic disorder, in the very dilute systems. This phenomenon is attested by the following features: a strong decrease of the solute-vacancy binding energy, and a smaller difference of the free energy barrier between the solute-vacancy and Fe-vacancy exchanges in the paramagnetic state. A significant increase of the correlation factor for the solute diffusion is also evidenced.

Further, the experimental and theoretical ratios of Mn diffusion coefficients to self-diffusion coefficients are presented and are in excellent agreement. The results show that relative to $\mathrm{Fe}, \mathrm{Mn}$ diffuses significantly faster in the ferromagnetic state as compared to the paramagnetic state.

\section{ACKNOWLEDGMENTS}

This work was partly supported by the French-German ANR-DFG MAGIKID Projects (No. DI 1419/14-1 and No. HI 1300/13-1). C.F. and A.S. thank DARI-GENCI resources under the A0090906020 Project and CINECA-MARCONI within the SISTEEL Project.

\section{APPENDIX}

\section{Parameters for EIM}

The parameters of the EIM are given in the following tables V-VIII, except for the Fe-Mn exchange-coupling parameter, which is not a constant (see Ref. [61] for details). Its expression is the following:

$$
\begin{aligned}
J_{\mathrm{Fe}-\mathrm{Mn}}^{n}= & {\left[J_{0}^{n} \cdot \frac{\theta-90^{\circ}}{90^{\circ}}\right]+a \cdot[M n]_{\mathrm{loc}}^{4} } \\
& +b \cdot[M n]_{\mathrm{loc}}^{3}+c \cdot[M n]_{\mathrm{loc}}^{2}+d \cdot[M n]_{\mathrm{loc}}+e,
\end{aligned}
$$

TABLE VIII. EIM parameters: Magnetic and chemical interaction parameters between a saddle point and a stable position (in $\mathrm{meV}$ ).

\begin{tabular}{lrr}
\hline \hline Distance & $1 n n$ & \multicolumn{1}{c}{$2 n n$} \\
\hline$J_{F e-F e}^{*}$ & -10.3 & 4.70 \\
$J_{M n-M n}^{*}$ & 1.51 & -1.30 \\
$J_{F e-M n}^{*}$ & 10.28 & -4.66 \\
$J_{M n-F e}^{*}$ & 5.60 & 3.99 \\
$V_{F e-F e}^{*}$ & -10.85 & 8.18 \\
$V_{M n-M n}^{*}$ & -4.63 & -1.93 \\
$V_{F e-M n}^{*}$ & -10.85 & 8.18 \\
$V_{M n-F e}^{*}$ & 2.97 & 0.01 \\
\hline \hline
\end{tabular}


where $[M n]_{\text {loc }}$ is the local Mn concentration in the five nearest-neighbor shells around the concerned atom and $\theta$ is the angle between the Mn magnetic moment and the average magnetic moment of the $\mathrm{Fe}$ atoms in the two nearest-neighbor shells of the concerned Mn atom (see Ref. [61]). The constant parameters of this expression are also presented in this Appendix.
[1] G. Hettich, H. Mehrer, and K. Maier, Scr. Metall. 11, 795 (1977).

[2] F. S. Buffington, K. Hirano, and M. Cohen, Acta Metall. 9, 434 (1961).

[3] C. M. Walter and N. L. Peterson, Phys. Rev. 178, 922 (1969).

[4] Y. Iijima, K. Kimura, and K. Hirano, Acta Metall. 36, 2811 (1988).

[5] D. W. James and G. M. Leak, Philos. Mag. 14, 701 (1966).

[6] R. J. Borg and C. E. Birchenall, Trans. AIME 218, 980 (1960).

[7] J. Geise and C. Herzig, Int. J. Mater. Res. 78, 291 (1987).

[8] D. Graham and D. H. Tomlin, Philos. Mag. 8, 1581 (1963).

[9] J. Kucera, B. Million, J. Ruzickova, V. Foldyna, and A. Jakobova, Acta Metall. 22, 135 (1974).

[10] G. R. Speich, J. A. Gula, and R. M. Fisher, The Electron Microprobe (Wiley, New York, 1966).

[11] S. J. Rothman, N. L. Peterson, C. M. Walter, and L. J. Nowicki, J. Appl. Phys. 39, 5041 (1968).

[12] V. A. Lazarev and V. M. Golikov, Phys. Metals Metallogr 29, 154 (1970).

[13] G. Salje and M. Feller-Knieppmeier, J. Appl. Phys. 48, 1833 (1977).

[14] Mehrer, H., Diffusion in Solids Fundamentals, Methods, Materials, Diffusion-Controlled Processes (Springer-Verlag, Berlin, 2007).

[15] M. Lübbehusen), Diploma work, University of Muenster, 1984.

[16] S. Huang, D. L. Worthington, M. Asta, V. Ozolins, G. Gosh, and P. K. Liaw, Acta Mater. 58, 1982 (2010).

[17] T. Nishizawa and K. Ishida, Binary Alloys Phase Diagrams 2 (ASM International, Materials Park, OH, 1990).

[18] I. Ohnuma, H. Enoki, O. Ikeda, R. Kainuma, H. Ohtani, B. Sundman, and K. Ishida, Acta Mater. 50, 379 (2002).

[19] T. Sourmail, Prog. Mater. Sci. 50, 816 (2005).

[20] A. V. Ruban and V. I. Razumovskiy, Phys. Rev. B 86, 174111 (2012).

[21] O. Senninger, E. Martínez, F. Soisson, M. Nastar, and Y. Bréchet, Acta Mater. 73, 97 (2014).

[22] D. Hobbs, J. Hafner, and D. Spisak, Phys. Rev. B 68, 014407 (2003).

[23] J. Hafner and D. Hobbs, Phys. Rev. B 68, 014408 (2003).

[24] D. Music, T. Takahashi, L. Vitos, C. Asker, I. A. Abrikosov, and J. M. Schneider, Appl. Phys. Lett. 91, 191904 (2007).

[25] V. T. Witusiewicz, F. Sommer, and E. J. Mittemeijer, J. Phase Equil. Diff. 25, 346 (2004)

[26] S. Bigdeli and M. Selleby, Calphad 64, 185 (2019).

[27] V. I. Anisimov, V. P. Antropov, A. I. Liechtenstein, V. A. Gubanov, and A. V. Postnikov, Phys. Rev. B 37, 5598 (1988).

[28] A. Schneider, C.-C. Fu, and C. Barreteau, Phys. Rev. B 98, 094426 (2018).

[29] H. Ding, V. I. Razumovskiy, and M. Asta, Acta Mater. 70, 130 (2014).

[30] N. Sandberg, Z. Chang, L. Messina, P. Olsson, and P. Korzhavyi, Phys. Rev. B 92, 184102 (2015).
[31] H. Wen and C. Woo, J. Nucl. Mater. 470, 102 (2016).

[32] O. Hegde, M. Grabowski, X. Zhang, O. Waseda, T. Hickel, C. Freysoldt, and J. Neugebauer, Phys. Rev. B 102, 144101 (2020),

[33] L. Ruch, D. Sain, H. Yeh, and L. Girifalco, J. Phys. Chem. Solids 37, 649 (1976).

[34] L. Girifalco, J. Phys. Chem. Solids 25, 323 (1964).

[35] R. J. Borg and D. Y. F. Lai, Acta Metall. 11, 861 (1963).

[36] C.-G. Lee, Y. Iijima, T. Hiratani, and K.-i. Hirano, Mater. Trans., JIM 31, 255 (1990).

[37] Y. Iijima, J. Phase Equilib. Diffus. 26, 466 (2005).

[38] K. Nohara and K.-i. Hirano, Suppl. Trans. Iron Steel Japan 11, 1267 (1971)

[39] J. S. Kirkaldy, P. N. Smith, and R. C. Sharma, Met. Trans. 4, 624 (1973).

[40] V. Irmer and M. Feller-Kniepmeier, J. Phys. Chem. Solids 33, 2141 (1972)

[41] L. Messina, M. Nastar, T. Garnier, C. Domain, and P. Olsson, Phys. Rev. B 90, 104203 (2014).

[42] C. D. Versteylen, N. H. van Dijk, and M. H. F. Sluiter, Phys. Rev. B 96, 094105 (2017).

[43] F. Kormann, B. Grabowski, B. Dutta, T. Hickel, L. Mauger, B. Fultz, and J. Neugebauer, Phys. Rev. Lett. 113, 165503 (2014).

[44] A. Schneider, C.-C. Fu, F. Soisson, and C. Barreteau, Phys. Rev. Lett. 124, 215901 (2020).

[45] F. Körmann, A. Dick, B. Grabowski, T. Hickel, and J. Neugebauer, Phys. Rev. B 85, 125104 (2012).

[46] J. Janssen, S. Surendralal, Y. Lysogorskiy, M. Todorova, T. Hickel, R. Drautz, and J. Neugebauer, Comput. Mater. Sci. 163, 24 (2019).

[47] N. Mousseau and G. T. Barkema, Phys. Rev. E 57, 2419 (1998).

[48] A. Zunger, S.-H. Wei, L.G. Ferreira, and J. E. Bernard, Phys. Rev. Lett. 65, 353 (1990).

[49] Z. S. Basinski, W. Hume-Rothery, and A. L. Sutton, Proc. R. Soc. London A 229, 459 (1955).

[50] C. Freysoldt, Comput. Mater. Sci. 133, 71 (2017).

[51] S. Boeck, C. Freysoldt, A. Dick, L. Ismer, and J. Neugebauer, Comput. Phys. Commun. 182, 543 (2011)

[52] J. P. Perdew, J. A. Chevary, S. H. Vosko, K. A. Jackson, M. R. Pederson, D. J. Singh, and C. Fiolhais, Phys. Rev. B 46, 6671 (1992).

[53] J. P. Perdew, K. Burke, and M. Ernzerhof, Phys. Rev. Lett. 77, 3865 (1996).

[54] P. E. Blochl, Phys. Rev. B 50, 17953 (1994).

[55] G. Kresse and D. Joubert, Phys. Rev. B 59, 1758 (1999).

[56] G. Kresse and J. Hafner, Phys. Rev. B 47, 558 (1993).

[57] G. Kresse and J. Furthmuller, Commun. Math. Phys. 6, 15 (1996).

[58] G. Kresse and J. Furthmuller, Phys. Rev. B 54, 11169 (1996).

[59] H. J. Monkhorst and J. D. Pack, Phys. Rev. B 13, 5188 (1976).

[60] M. Methfessel and A. T. Paxton, Phys. Rev. B 40, 3616 (1989).

[61] A. Schneider, C.-C. Fu, O. Waseda, C. Barreteau, and T. Hickel, Phys. Rev. B 103, 024421 (2021). 
[62] M. Y. Lavrentiev, D. Nguyen-Manh, and S. L. Dudarev, Phys. Rev. B 81, 184202 (2010).

[63] M. Y. Lavrentiev, J. S. Wrobel, D. Nguyen-Manh, and S. L. Dudarev, Phys. Chem. Chem. Phys. 16, 16049 (2014).

[64] M. Y. Lavrentiev, J. S. Wrobel, D. Nguyen-Manh, S. L. Dudarev, and M. G. Ganchenkova, J. Appl. Phys. 120, 043902 (2016).

[65] A. V. Ruban, S. Khmelevskyi, P. Mohn, and B. Johansson, Phys. Rev. B 75, 054402 (2007).

[66] F. Keffer, Handbuch der Physik, 18, pt. 2 (Springer-Verlag, New York, 1966).

[67] F. Soisson and C. C. Fu, Phys. Rev. B 76, 214102 (2007).

[68] E. Martinez, O. Senninger, C.-C. Fu, and F. Soisson, Phys. Rev. B 86, 224109 (2012).

[69] Y. Chen, C. L. Fu, K. M. Ho, and B. N. Harmon, Phys. Rev. B 31, 6775 (1985).

[70] C. Wolverton, V. Ozolins, and M. Asta, Phys. Rev. B 69, 144109 (2004).

[71] A. Togo and I. Tanaka, Scr. Mater. 108, 1 (2015).

[72] A. Einstein et al., Ann. phys. 322, 549 (1905).

[73] A. Einstein, Ann. phys. 324, 371 (1906).

[74] M. Von Smoluchowski, Ann. Phys. 326, 756 (1906).

[75] Y. Adda and J. Phillibert, Nat. Sci. 1, 264 (1966).

[76] A. D. Le Claire, J. Nucl. Mater. 69, 70 (1978).

[77] For comparison, a similar mixing scheme was used for the diffusion coefficients instead of migration barriers and the resulting ratios were very similar.

[78] H. Takeuchi, Y. Yogo, T. Hattori, T. Tajima, and T. Ishikawa, ISIJ Int. 57, 1883 (2017).
[79] L. Bergqvist and A. Bergman, Phys. Rev. Mater. 2, 013802 (2018).

[80] C. H. Woo, H. Wen, A. A. Semenov, S. L. Dudarev, and P. W. Ma, Phys. Rev. B 91, 104306 (2015).

[81] Y. Mishin, J. Schimmelpfennig, and G. Borchardt, J. Phys. (Paris) 7, 1797 (1997).

[82] G. E. Murch and S. J. Rothman, Philos. Mag. A. 43, 229 (1981).

[83] F. Wenwer, A. Gude, G. Rummel, M. Eggersmann, T. Zumkley, N. Stolwijk, and H. Mehrer, Meas. Sci. Technol. 7, 632 (1996).

[84] L. Harrison, Trans. Faraday Soc. 57, 1191 (1961).

[85] A. Le Claire, Br. J. Appl. Phys. 14, 351 (1963).

[86] Paul, A. and Laurila, T. and Vuorinen, V. and Divinski, S.V., Thermodynamics, Diffusion and the Kirkendall Effect in Solids (Springer International Publishing, Switzerland, 2014).

[87] L. Messina, M. Nastar, N. Sandberg, and P. Olsson, Phys. Rev. B 93, 184302 (2016).

[88] O. I. Gorbatov, P. A. Korzhavyi, A. V. Ruban, B. Johansson, and Y. N. Gornostyrev, J. Nucl. Mater. 419, 248 (2011).

[89] J. Crangle and G. Goodman, Proc. Roy. Soc. London A 321, 477 (1971).

[90] P. Klugkist and C. Herzig, Phys. Stat. Sol. 148, 413 (1995).

[91] J. Kucera, B. Million, and J. Ruzickova, Phys. Stat. Solidi A 96, 177 (1986).

[92] Y. Iijima, K. Kimura, C. Lee, and K. Hirano, Mater. Trans. JIM 34, 20 (1993).

[93] H. Mehrer, D. Hoepfel, and G. Hettich, DIMETA 82, Diffusion in Metals and Alloys (Trans Tech Publications, Switzerland, 1983). 